\title{
A MEASUREMENT OF THE COSMIC MICROWAVE BACKGROUND GRAVITATIONAL LENSING POTENTIAL FROM 100 SQUARE DEGREES OF SPTPOL DATA
}

K. T. Story ${ }^{1,2}$, D. Hanson ${ }^{3}$, P. A. R. Ade ${ }^{4}$, K. A. Aird ${ }^{5}$, J. E. Austermann ${ }^{6}$, J. A. Beall ${ }^{7}$, A. N. Bender ${ }^{3,8}$, B. A. Benson ${ }^{1,9,10}$, L. E. Bleem ${ }^{1,2,8}$, J. E. Carlstrom ${ }^{1,2,8,9,11}$, C. L. Chang ${ }^{1,8,9}$, H. C. Chiang ${ }^{12}$, H-M. Cho ${ }^{13}$, R. Citron ${ }^{1}$, T. M. Crawford ${ }^{1,9}$, A. T. Crites ${ }^{1,9,14}$, T. de HaAn ${ }^{15}$, M. A. Dobbs ${ }^{3,16}$, W. Everett ${ }^{6}$, J. Gallichio ${ }^{1}$, J. Gao ${ }^{7}$, E. M. George ${ }^{15}$, A. Gilbert ${ }^{3}$, N. W. Halverson ${ }^{6,17}$, N. Harrington ${ }^{15}$, J. W. Henning ${ }^{1,6}$, G. C. Hilton ${ }^{7}$, G. P. Holder ${ }^{3}$, W. L. Holzapfel ${ }^{15}$, S. Hoover ${ }^{1,2}$, Z. Hou ${ }^{1}$, J. D. Hrubes ${ }^{5}$, N. Huang ${ }^{15}$, J. Hubmayr ${ }^{7}$, K. D. Irwin ${ }^{13,18}$, R. Keisler ${ }^{18,19}$, L. KNOX ${ }^{20}$, A. T. LeE $^{15,21}$, E. M. LeitCH ${ }^{1,9}$,

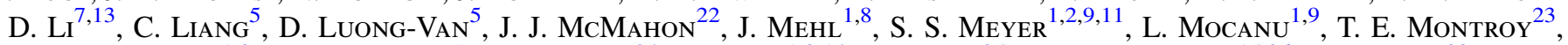
T. Natoli ${ }^{1,2}$, J. P. Nibarger ${ }^{7}$, V. Novosad ${ }^{24}$, S. PAdin ${ }^{1,9,14}$, C. Pryke ${ }^{25}$, C. L. Reichardt ${ }^{15,26}$, J. E. RuhL ${ }^{23}$,

B. R. Saliwanchik ${ }^{23}$, J. T. SAYre ${ }^{23}$, K. K. Schaffer ${ }^{1,11,27}$, G. Smecher ${ }^{3,28}$, A. A. Stark ${ }^{29}$, C. Tucker ${ }^{4}$, K. VAnderlinde ${ }^{30,31}$, J. D. VieIra ${ }^{32,33}$, G. WANG ${ }^{8}$, N. Whitehorn ${ }^{15}$, V. YeFremenko ${ }^{8}$, and O. ZahN ${ }^{34}$

${ }^{1}$ Kavli Institute for Cosmological Physics, University of Chicago, 5640 South Ellis Avenue, Chicago, IL 60637, USA; kstory@uchicago.edu

${ }^{2}$ Department of Physics, University of Chicago, 5640 South Ellis Avenue, Chicago, IL 60637, USA

${ }^{3}$ Department of Physics, McGill University, 3600 Rue University, Montreal, Quebec H3A 2T8, Canada ${ }^{4}$ Cardiff University, Cardiff CF10 3XQ, UK

${ }^{5}$ University of Chicago, 5640 South Ellis Avenue, Chicago, IL 60637, USA

${ }^{6}$ Department of Astrophysical and Planetary Sciences, University of Colorado, Boulder, CO 80309, USA

NIST Quantum Devices Group, 325 Broadway Mailcode 817.03, Boulder, CO 80305, USA

${ }^{8}$ High Energy Physics Division, Argonne National Laboratory, 9700 S. Cass Avenue, Argonne, IL 60439, USA

${ }^{9}$ Department of Astronomy and Astrophysics, University of Chicago, 5640 South Ellis Avenue, Chicago, IL 60637, USA

${ }^{10}$ Fermi National Accelerator Laboratory, MS209, P.O. Box 500, Batavia, IL 60510, USA

${ }^{11}$ Enrico Fermi Institute, University of Chicago, 5640 South Ellis Avenue, Chicago, IL 60637, USA

${ }^{12}$ School of Mathematics, Statistics \& Computer Science, University of KwaZulu-Natal, Durban, South Africa

${ }^{13}$ SLAC National Accelerator Laboratory, 2575 Sand Hill Road, Menlo Park, CA 94025, USA

${ }^{14}$ California Institute of Technology, MS 249-17, 1216 E. California Blvd., Pasadena, CA 91125, USA

${ }^{15}$ Department of Physics, University of California, Berkeley, CA 94720, USA

${ }^{16}$ Canadian Institute for Advanced Research, CIFAR Program in Cosmology and Gravity, Toronto, ON, M5G 1Z8, Canada

${ }^{17}$ Department of Physics, University of Colorado, Boulder, CO 80309, USA

${ }^{18}$ Department of Physics, Stanford University, 382 Via Pueblo Mall, Stanford, CA 94305, USA

${ }^{19}$ Kavli Institute for Particle Astrophysics and Cosmology, Stanford University, 452 Lomita Mall, Stanford, CA 94305, USA

${ }^{20}$ Department of Physics, University of California, One Shields Avenue, Davis, CA 95616, USA

${ }^{21}$ Physics Division, Lawrence Berkeley National Laboratory, Berkeley, CA 94720, USA

${ }^{22}$ Department of Physics, University of Michigan, 450 Church Street, Ann Arbor, MI 48109, USA

${ }^{23}$ Physics Department, Center for Education and Research in Cosmology and Astrophysics, Case Western Reserve University, Cleveland, OH 44106, USA

${ }^{24}$ Materials Sciences Division, Argonne National Laboratory, 9700 S. Cass Avenue, Argonne, IL 60439, USA

${ }^{25}$ School of Physics and Astronomy, University of Minnesota, 116 Church Street S.E. Minneapolis, MN 55455, USA

${ }^{26}$ School of Physics, University of Melbourne, Parkville, VIC 3010, Australia

${ }^{27}$ Liberal Arts Department, School of the Art Institute of Chicago, 112 S Michigan Ave, Chicago, IL 60603, USA

${ }^{29}$ Harvard-Smithsonian Center for Astrophysics, 60 Garden Street, Cambridge, MA 02138, USA

${ }^{30}$ Dunlap Institute for Astronomy \& Astrophysics, University of Toronto, 50 St. George Street, Toronto, ON, M5S 3H4, Canada

${ }^{31}$ Department of Astronomy \& Astrophysics, University of Toronto, 50 St. George Street, Toronto, ON, M5S 3H4, Canada

Astronomy Department, University of Illinois at Urbana-Champaign, 1002 W. Green Street, Urbana, IL 61801, USA

${ }^{33}$ Department of Physics, University of Illinois Urbana-Champaign, $1110 \mathrm{~W}$. Green Street, Urbana, IL 61801, USA

${ }^{34}$ Berkeley Center for Cosmological Physics, Department of Physics, University of California, and Lawrence Berkeley National Laboratory, Berkeley, CA 94720, USA

Received 2014 December 15; accepted 2015 June 12; published 2015 August 28

\begin{abstract}
We present a measurement of the cosmic microwave background (CMB) gravitational lensing potential using data from the first two seasons of observations with SPTpol, the polarization-sensitive receiver currently installed on the South Pole Telescope. The observations used in this work cover $100 \mathrm{deg}^{2}$ of sky with arcminute resolution at $150 \mathrm{GHz}$. Using a quadratic estimator, we make maps of the CMB lensing potential from combinations of CMB temperature and polarization maps. We combine these lensing potential maps to form a minimum-variance (MV) map. The lensing potential is measured with a signal-to-noise ratio of greater than one for angular multipoles between $100<L<250$. This is the highest signal-to-noise mass map made from the CMB to date and will be powerful in cross-correlation with other tracers of large-scale structure. We calculate the power spectrum of the lensing potential for each estimator, and we report the value of the MV power spectrum between $100<L<2000$ as our primary result. We constrain the ratio of the spectrum to a fiducial $\Lambda \mathrm{CDM}$ model to be $A_{\mathrm{MV}}=0.92 \pm 0.14$ (Stat.) \pm 0.08 (Sys.). Restricting ourselves to polarized data only, we find $A_{\mathrm{POL}}=0.92 \pm 0.24$ (Stat.) \pm 0.11 (Sys.). This measurement rejects the hypothesis of no lensing at $5.9 \sigma$ using polarization data alone, and at $14 \sigma$ using both temperature and polarization data.
\end{abstract}

Key words: cosmic background radiation - cosmology: observations - large-scale structure of universe 


\section{INTRODUCTION}

Gravitational lensing of the cosmic microwave background (CMB) has become a powerful observational tool for probing the geometry of the universe and the growth of large-scale structure. As light travels through the universe, the paths of photons are bent by gravitational interactions with matter. These deflections encode information about the growth of large-scale structure at late times, when massive neutrinos suppress structure growth and dark energy drives the accelerated expansion of the universe. As a result, lensing measurements provide a valuable method for constraining neutrino masses (Lesgourgues \& Pastor 2006) and dark energy (Calabrese et al. 2009). The CMB provides a unique source for lensing measurements: the $\mathrm{CMB}$ is well characterized, originated at a well known redshift, and has traversed nearly the entire observable universe, in principle providing information about all of the structure between Earth and the last scattering surface of the CMB. The lensing signal is sourced from a broad range of redshifts, typically $0.1<z<5$ (Lewis $\&$ Challinor 2006).

We can measure gravitational lensing by looking for distortions in the characteristic patterns of the primordial CMB temperature and polarization (Blanchard \& Schneider 1987; for a review, see Lewis \& Challinor 2006). The CMB is partially polarized, and can be decomposed into evenparity ( $E$-mode) and odd-parity $(B$-mode) components (e.g., Hu \& White 1997). Lensing stretches and contracts observed $\mathrm{CMB}$ anisotropies. These distortions smooth the $\mathrm{CMB}$ temperature (Seljak 1996) and $E$-mode power spectra, produce lensed $B$-mode power on scales where there is very little primordial power (Zaldarriaga \& Seljak 1998), and correlate modes that were originally independent (Cole \& Efstathiou 1989). Lensing in the CMB was first detected using lensing-galaxy correlations (Smith et al. 2007; Hirata et al. 2008). The smoothing of the CMB power spectrum has subsequently been well measured in temperature (Story et al. 2013; Das et al. 2014; Planck Collaboration XVI 2014) and should soon be detected in the $E$-mode power spectrum.

Lensing-induced $B$-modes were first detected in crosscorrelation using data from SPTpol, the polarization-sensitive receiver currently installed on the South Pole Telescope (SPT, Carlstrom et al. 2011); SPTpol data was cross-correlated with the cosmic infrared background, resulting in a $7.7 \sigma$ detection of $B$-modes (Hanson et al. 2013, hereafter H13). Using similar cross-correlation analyses, POLARBEAR found $2.3 \sigma$ evidence for lensed $B$-modes (POLARBEAR Collaboration 2014b), and the Atacama Cosmology Telescope Polarimeter (ACTPol) collaboration measured lensed $B$-modes with a $3.2 \sigma$ significance (van Engelen et al. 2014b). Recently, POLARBEAR made a measurement of the $B$-mode auto-spectrum which disfavored the no-lensing hypothesis at $2.0 \sigma$ (POLARBEAR Collaboration 2014a), and BICEP2 measured a $B$-mode autospectrum which contains a lensing component measured at 5.5 $\sigma$ (BICEP2 Collaboration 2014).

The correlation between initially independent CMB modes introduced by lensing can be measured using a quadratic estimator technique (Seljak \& Zaldarriaga 1999; Hu 2001; Hu \& Okamoto 2002). With this technique, the amplitude of the lensing potential power spectrum has been measured to high precision using CMB temperature data: $22 \%$ using data from $\sim 600 \mathrm{deg}^{2}$ of sky observed with the Atacama Cosmology Telescope (ACT; Das et al. 2014), 19\% using data from $\sim 590 \mathrm{deg}^{2}$ of the $2500 \mathrm{deg}^{2}$ SPT-SZ survey observed with the SPT (van Engelen et al. 2012), and 4\% using data from $\sim 28,000 \mathrm{deg}^{2}$ of sky (70\% of the full sky) observed with Planck (Planck Collaboration XVII 2013). ${ }^{35}$ Data from the full $2500 \mathrm{deg}^{2}$ SPT-SZ survey is expected to yield a measurement of the lensing amplitude with a significance similar to that of the current Planck result. POLARBEAR has detected gravitational lensing with a quadratic-estimator technique using only estimators that include $B$-modes, rejecting the no-lensing hypothesis at $4.2 \sigma$ over $30 \mathrm{deg}^{2}$ (POLARBEAR Collaboration 2014c); combining this with their $B$-mode auto-spectrum measurement, POLARBEAR rejected the no-lensing hypothesis at $4.7 \sigma$ (POLARBEAR Collaboration 2014a).

In this paper, we construct a map of the gravitational lensing potential and its power spectrum from temperature and polarization data collected with SPTpol within a $100 \mathrm{deg}^{2}$ patch of the southern sky. We combine the temperature and polarization measurements into a minimum-variance (MV) estimate of the lensing potential.

Throughout this paper, we use a fiducial $\Lambda \mathrm{CDM}$ cosmological model that provides the best fit to the combination of Planck power spectrum, Planck lensing spectrum, WMAPpolarization, SPT, and ACT data (Planck Collaboration XVI 2014), with the following parameters: baryon density $\Omega_{b} h^{2}=0.0222$, cold dark matter density $\Omega_{c} h^{2}=0.1185$, Hubble parameter $H_{0}=100 h \mathrm{~km} \mathrm{~s}^{-1} \mathrm{Mpc}^{-1}$ with $h=$ 0.6794 , power spectrum of primordial curvature perturbations with an amplitude (at $k=0.05 \mathrm{Mpc}^{-1}$ ) $A_{s}=2.21 \times 10^{-9}$ and spectral index $n_{s}=0.9624$, optical depth to reionization $\tau=0.0943$, and a neutrino energy density corresponding to a sum over the neutrino mass eigenstates of $0.06 \mathrm{eV}$. Hereafter, this model will be referred to as the "PLANCK+LENS+WP +HIGHL" model. This fiducial model serves as the input to our simulations and the reference for calculating the amplitude of the lensing potential power spectrum.

This paper is organized as follows: we describe the data used in this paper in Section 2. We review the theoretical description of gravitational lensing and describe our implementation of the quadratic estimator in Section 3. We describe the simulations in Section 4. We detail our uncertainty budget in Section 5. We present our results in Section 6. We discuss potential sources of systematic errors in Section 7. We discuss the implications of this measurement in Section 8.

\section{SPTPOL OBSERVATIONS AND DATA-PROCESSING}

This paper uses maps of the CMB temperature and polarization made with data from SPTpol (Austermann et al. 2012). SPTpol is a polarization-sensitive receiver that was installed in early 2012 on the $10 \mathrm{~m}$ SPT (Carlstrom et al. 2011). We use data obtained from observations during 2012 and a few months of 2013. The 2012 data have already been used to make the first detection of lensing $B$ modes (H13) and to make the most precise measurement of the high $\ell E$ mode power spectrum and temperature- $E$-mode cross-spectrum to date (Crites et al. 2014, hereafter C14). A more thorough discussion of the SPTpol instrument and data set is given in C14; here we summarize the most important properties of the SPTpol data and resulting maps.

\footnotetext{
35 The fractional precision is reported relative to the mean of each measurement.
} 
The SPTpol receiver contains 1536 polarization-sensitive transition edge sensor bolometers, with 1176 detectors at $150 \mathrm{GHz}$ and 360 detectors at $95 \mathrm{GHz}$. In this analysis we use only the $150 \mathrm{GHz}$ data. During 2012 and the first part of 2013, SPTpol was used to observe the $100 \mathrm{deg}^{2}$ "SPTpol deep field," a roughly square patch of sky spanning R.A. of 23-24 hr and decl. of $-50^{\circ}$ to $-60^{\circ}$. The data consists of 5182 individual field observations, each lasting approximately 30 minutes. The observations were performed using a lead-trail observing strategy; the field is split into two halves ("lead" and "trail") in R.A., which are scanned separately such that each half-field is observed over the same range in azimuth. The half-fields are observed by scanning the telescope back and forth in azimuth, followed by a small step in elevation; this process is repeated until the entire half-field is covered. We refer to a single sweep of the telescope from one side of the field to the other as a "scan," and to a set of scans that cover the entire half-field as an "observation." The lead-trail strategy allows the subtraction of ground pickup by differencing pairs of lead and trail observations, however, in this work we simply coadd lead and trail observations into single observation maps.

The raw field observations consist of time-ordered data (TOD) for each SPTpol bolometer. The individual bolometer TOD are calibrated relative to one another using a combination of regular calibration observations of an internal chopped blackbody source and of the galactic $\mathrm{H}_{\mathrm{II}}$ region $\mathrm{RCW} 38$. The absolute calibration $\left(T_{\text {cal }}\right)$ is tied to the CMB maps produced by Planck. The absolute temperature calibration uncertainty is estimated to be $\delta_{\mathrm{Tcal}}=1.3 \%$. See $\mathrm{C} 14$ for a detailed description of this calibration process.

The TOD are filtered before being accumulated into maps. To suppress atmospheric fluctuations, a fourth-order polynomial is subtracted from the TOD of each detector for every scan. A low-pass filter is also applied to prevent aliasing at the pixelization scale, with a cutoff corresponding roughly to a maximum angular scale of $\ell \sim 4000(\sim 3 \mathrm{~Hz}$ at the telescope scan-speed).

Maps are made by calculating the Stokes parameters $I, Q$, and $U$ in each map pixel. We refer to the Stokes parameter $I$ as $T$ (temperature), and use temperature units for our $T, Q$, and $U$ maps. The TOD are accumulated into maps using the pointing, polarization angle, and efficiency of each detector, as well as a weight calculated from the noise power of each detector between 1 and $3 \mathrm{~Hz}(1300 \lesssim \ell \lesssim 3900$ at the telescope scanspeed). The maps are pixelized in an oblique Lambert azimuthal equal-area projection, with square $2^{\prime} \times 2^{\prime}$ pixels.

The $Q$ and $U$ maps are multiplied by an additional calibration factor $P_{\text {cal }}$ to correct for errors in the measured detector polarization calibration and efficiency. We use a value of $P_{\text {cal }}=1.048$ from Table 3 in C14. This value is calculated using a Markov Chain Monte Carlo (MCMC) ${ }^{36}$ by fitting the SPTpol EE and TE bandpowers jointly with Planck TT bandpowers (Planck Collaboration XVI 2014) and WMAP9 polarization data (Hinshaw et al. 2013) in a $\Lambda$ CDM model with varying nuisance parameters; see $\mathrm{C} 14$ for details. The value of $P_{\text {cal }}$ for the best-fit model from this MCMC-chain is taken as our estimate of the polarization calibration parameter. The $P_{\mathrm{cal}}$ calibration has an uncertainty of $\delta_{\text {Pcal }}=1.7 \%$, which is calculated from the marginalized posterior of $P_{\text {cal }}$. We treat $\delta_{\text {Pcal }}$ as uncorrelated with other systematic uncertainties in

\footnotetext{
${ }^{36}$ We calculate the MCMC chain using the CosmoMC package (Lewis \& Bridle 2002).
}

Section 5; this over-estimates the uncertainty since $P_{\text {cal }}$ is partially correlated with other parameters in the fit (e.g., $\left.T_{\text {cal }}\right)$.

The final SPTpol deep field maps have nearly homogeneous coverage, with inverse-noise-weighted pixel hit counts varying by less than $\sim 35 \%$ over the $100 \mathrm{deg}^{2}$ field (corresponding to the difference in the cosine of the declination angle between the top and bottom of the field), except for a $\sim 2 \times$ deeper strip at the center caused by overlap of the lead and trail fields. The maps have an effective noise level, estimated between $2000<\ell<3000$, of $\sim 11 \mu \mathrm{K}$ arcmin in temperature and $\sim 9$ $\mu \mathrm{K}$ arcmin in $Q$ and $U$ (atmospheric noise causes a higher noise level in $T$ than in $Q$ or $U$ ). We use data-quality checks to cut data at three stages: individual bolometers, scans, and observations. These cuts are described in detail in C14. All data that pass these cuts are coadded into final $T, Q$, and $U$ maps.

Systematic effects such as detector gain errors can cause temperature power to leak into the $Q$ and $U$ polarization maps. We correct for the leakage by subtracting the appropriately scaled temperature map from each polarization map as follows: $Q=Q_{\text {raw }}-\hat{\epsilon}^{Q} T$, and an analogous expression for $U$. We find $\hat{\epsilon}^{Q}=+0.0050$ and $\hat{\epsilon}^{U}=-0.0083$. See C14 for more detail.

Signal power in the final maps is suppressed by two processes: data filtering and the telescope angular response function, or beam. We model the signal power that is removed by filtering as a $2 \mathrm{D}$ Fourier-space ${ }^{37}$ transfer function $F_{\ell}^{\text {filt }}$. We obtain this transfer function using simulations of the filtering process. The SPT beam suppresses power on arcminute scales. We approximate this beam as radially symmetric, with a $2 \mathrm{D}$ Fourier-space transfer function $F_{\ell}^{\text {beam }}$ measured using observations of Mars as well as bright point sources in the deep field. The beam was measured independently in both 2012 and 2013; we use a composite beam calculated as the inverse-noisevariance weighted average of the beams from each year. Taken together, we model the total transfer function as

$$
F_{\ell}^{\text {tot }}=F_{\ell}^{\text {pix }} F_{\ell}^{\text {filt }} F_{\ell}^{\text {beam }}
$$

where $F_{\ell}^{\text {pix }}$ is the 2D Fourier transform of a square $2^{\prime}$ pixel.

The SPTpol deep field contains a number of bright point sources, which we mask. We identify all sources detected at $>5 \sigma$ at $150 \mathrm{GHz}$ from Mocanu et al. (2013), which corresponds to a flux-cut of approximately $6 \mathrm{mJy}$; all pixels within $5^{\prime}$ of each source are masked in both the TOD filtering and the final maps. We extend this mask to $10^{\prime}$ for all very bright sources detected at $>75 \sigma$. We also use a $10^{\prime}$ radius to mask all galaxy clusters detected in this field by Vanderlinde et al. (2010) using the Sunyaev-Zel'dovich effect. This masking removes approximately 120 sources, cutting $5 \mathrm{deg}^{2}$ of the field. We additionally multiply the maps by a sky mask that down-weights the noisy edges of the maps.

The processing described in this section results in a set of three coadded, masked maps: $T(\hat{\boldsymbol{n}}), Q(\hat{\boldsymbol{n}})$, and $U(\hat{\boldsymbol{n}})$. These maps are filtered with the $C$-inverse filtering process (see Section 3.1), which transforms the set of maps into filtered Fourier-space maps $\bar{T}_{\ell}, \bar{E}_{\ell}$, and $\bar{B}_{\ell}$.

We apply two cuts in Fourier-space. The data maps have noise that arises from two contributions: a roughly isotropic component from atmosphere at low $|\ell|$ and an anisotropic component from low-frequency noise that is uncorrelated

\footnotetext{
37 Throughout this paper, we adopt the flat-sky approximation, where wavenumbers are represented by the discretized two-dimensional vector $\ell$, the magnitude of $\ell$ is equivalent to multipole number $\ell$, and spherical harmonic transforms are replaced by Fourier transforms.
} 
between detectors at low $\left|\ell_{x}\right|$. We remove these noisy regions of Fourier space, keeping only modes with $\left|\ell_{x}\right|>450$. Second, we use only modes with $|\ell|<3000$ when estimating the lensing potential. We verify that our results are not sensitive to the exact choices of these cuts; see Section 7 for details.

Thus the data input to the estimator of the lensing potential (i.e., Equation (9)) is a set of three filtered maps: $\bar{T}_{\ell}, \bar{E}_{\ell}$, and $\bar{B}_{\ell}$.

\section{CMB LENSING ANALYSIS}

As CMB photons travel through the universe, their paths are deflected by gravitational interactions with intervening largescale structure. These deflections can be described as a remapping of the $\mathrm{CMB}$ temperature and polarization fields (Lewis \& Challinor 2006):

$$
\begin{aligned}
T(\hat{\boldsymbol{n}}) & =\tilde{T}(\hat{\boldsymbol{n}}+\nabla \phi(\hat{\boldsymbol{n}})) \\
{[Q \pm i U](\hat{\boldsymbol{n}}) } & =[\tilde{Q} \pm i \tilde{U}](\hat{\boldsymbol{n}}+\nabla \phi(\hat{\boldsymbol{n}})),
\end{aligned}
$$

where $\hat{\boldsymbol{n}}$ is a unit-vector denoting a particular line of sight direction on the sky. Throughout the paper, tildes denote unlensed quantities. The projected lensing potential $\phi(\hat{\boldsymbol{n}})$ is an integral over the three-dimensional potential $\Psi\left(\chi \hat{\boldsymbol{n}} ; \eta_{0}-\chi\right)$ :

$$
\phi(\hat{\boldsymbol{n}})=-2 \int_{0}^{\chi_{\mathrm{CMB}}} d \chi \frac{f_{K}\left(\chi_{\mathrm{CMB}}-\chi\right)}{f_{K}\left(\chi_{\mathrm{CMB}}\right) f_{K}(\chi)} \Psi\left(\chi \hat{\boldsymbol{n}} ; \eta_{0}-\chi\right) .
$$

where $\chi$ is the comoving distance along the line of sight, $\chi_{\mathrm{CMB}}$ is the comoving distance to the surface of last scattering, the quantity $\eta_{0}-\chi$ is the conformal time at which a CMB photon would have been at position $\chi \hat{\boldsymbol{n}}$, and $f_{K}(\chi)$ is the comoving angular distance with $f_{K}(\chi)=\chi$ in a flat universe. The deflection field of CMB photons is given by $\boldsymbol{d}(\hat{\boldsymbol{n}})=\nabla \phi(\hat{\boldsymbol{n}})$. The apparent local expansion or contraction of the $\mathrm{CMB}$ is given by the divergence of $\nabla \phi$; the convergence field is defined as $\kappa(\hat{\boldsymbol{n}}) \equiv-\frac{1}{2} \nabla^{2} \phi(\hat{\boldsymbol{n}})$. In this paper, final maps and power spectra will be presented in terms of $\kappa$.

The unlensed CMB sky is well described as a statisticallyisotropic, Gaussian random field; in this case, all statistical information about the field is contained in the power spectrum

$$
C_{\ell}^{X Y}=\delta\left(\ell-\ell^{\prime}\right)\left\langle X_{\ell} Y_{\ell^{\prime}}^{*}\right\rangle
$$

where $X, Y \in[T, E, B]$ are harmonic-space CMB maps. Gravitational lensing introduces statistical anisotropy in the CMB temperature and polarization fields, correlating multipole moments across a range defined by the lensing deflection field $\boldsymbol{d}(\hat{\boldsymbol{n}})$ (Hu \& Okamoto 2002). Averaging over realizations of the lensed $\mathrm{CMB}$ fields for a fixed lensing potential, we can expand the covariance of CMB fields in a Taylor series as a function of $\phi$ (Hu \& Okamoto 2002):

$$
\begin{aligned}
\left\langle X_{\ell} Y_{\ell^{\prime}}^{*}\right\rangle_{\mathrm{CMB}}= & \delta\left(\ell-\ell^{\prime}\right) C_{l}^{X Y} \\
& +W_{\ell, \ell^{\prime}}^{\phi^{X Y}} \phi_{\ell-\ell^{\prime}}+\mathcal{O}\left(\phi^{2}\right),
\end{aligned}
$$

where $W_{\ell, \ell^{\prime}}^{\phi^{X Y}}$ is the coefficient on the $\mathcal{O}(\phi)$ term. Information about the lensing potential is thus contained in the off-diagonal elements $\left(\ell \neq \ell^{\prime}\right)$ of the second term and subsequent higher order terms. Said another way, lensing distorts the observed CMB anisotropies, which introduces statistical anisotropy in the covariance of the $\mathrm{CMB}$; these changes are encapsulated in the second and higher-order terms in Equation (5).

The analysis in this paper will proceed in the following manner: first we filter the $\mathrm{CMB}$ fields in order to maximize signal to noise for the lensing calculation. Next we estimate the two-dimensional lensing potential $\phi$ from these filtered fields. We correct $\phi$ for two effects: a multiplicative normalization and an additive "mean-field" (MF) bias correction. Then we calculate the power spectrum of $\phi$, and subtract two noisebias terms. Finally, we calculate the amplitude of the final spectrum relative to a fiducial cosmology.

\subsection{Map Filtering}

The first step in this analysis is to filter the CMB fields to maximize the $\mathrm{CMB}$ signal relative to the noise. We use the following data model to motivate our filtering choices. We will refer to data maps in position-space as $d_{j}$, where $d \in[T(\hat{\boldsymbol{n}}), Q(\hat{\boldsymbol{n}}), U(\hat{\boldsymbol{n}})]$ and $j$ is the pixel index. We assume that these data maps are a combination of the sky signal $X_{\ell} \in\left[T_{\ell}, E_{\ell}, B_{\ell}\right]$ and noise given by

$$
d_{j}=\sum_{\ell} P_{j \ell} X_{\ell}+\sum_{\ell} P_{j \ell} N_{\ell}+n_{j}
$$

Here, $P_{j \ell}$ is a matrix operator that applies any filter transfer functions (for example, smoothing by the instrumental beam, pixelization, and timestream filtering, e.g., Equation (1)) and inverse-Fourier transforms the sky signal. Additionally, $P_{j \ell}$ transforms $E_{\ell}$ and $B_{\ell}$ into $Q(\hat{\boldsymbol{n}})$ and $U(\hat{\boldsymbol{n}})$.

The map noise is modeled with two components. The Fourier-domain noise contribution $N_{\ell}$ represents "sky noise" that comes from components of the sky that do not trace the $\mathrm{CMB}$, such as foregrounds or emission from the atmosphere. The map-domain noise contribution $n_{j}$ arises from instrumental noise; it is assumed to be white and uncorrelated between pixels. For modes that are heavily filtered, the instrumental noise may be rolled off just as the sky signal is. We ignore this effect, with the understanding that it causes our inverse-variance filtering procedure to overestimate the instrumental variance on such scales (relative to the sky component, which does include a filter-transfer function). Over-estimating the instrumental variance in the filter is slightly sub-optimal but will not bias the CMB filtered fields.

We now build a filter tuned to maximize signal-to-noise of CMB anisotropy Fourier modes. This is essentially a "matched filter" technique using an inverse-variance filter for data maps that have anisotropic noise and a filter transfer function. With a map $d_{j}$ described by Equation (6), we can determine inversevariance filtered Fourier modes $\bar{X}$ as

$$
\left[S^{-1}+P^{\dagger} n^{-1} P\right] S \bar{X}=P^{\dagger} n^{-1} d .
$$

We solve for $\bar{X}$, using a conjugate-gradient-descent method to evaluate the expression

$$
\bar{X}=S^{-1}\left[S^{-1}+P^{\dagger} n^{-1} P\right]^{-1} P^{\dagger} n^{-1} d,
$$

where throughout this paper, we will use an over-bar notation for inverse-variance-weighted quantities. Here $S$ is the total sky signal covariance matrix, defined as the sum of the signal and "sky noise" power: $S \equiv C_{\ell}^{X}+C_{\ell}^{N}$; in this expression, $C_{\ell}^{X}$ is the theoretical power spectrum of $X$ evaluated on the 2D Fourier 
plane, and $C_{\ell}^{N}$ is the $2 \mathrm{D}$ power spectrum of $N_{\ell}$ from Equation (6), specifically, $\left\langle\left|N_{\ell}\right|^{2}\right\rangle$. The matrix $n^{-1}$ is the sky mask multiplied by the inverse of the map-noise variance $\left\langle\left|n_{j}^{2}\right|\right\rangle$. Specifically, $n$ is calculated by taking the map-noise $n_{j}$ from Equation (6) then setting the inverse-noise level $n_{j}^{-1}=0$ for masked pixels $j$ (this is equivalent to taking $n_{j} \rightarrow \infty$ for masked pixels).

The filtered fields $\bar{X}_{\ell}$ form the input to the estimator of the lensing potential.

\subsection{Quadratic Estimator of $\phi$}

We now construct an estimator for the lensing potential that takes advantage of the off-diagonal correlations introduced by lensing in the CMB fields (Hu \& Okamoto 2002). Calculating a properly normalized, unbiased estimate of $\phi$ requires three steps that are detailed below:

1. Calculate an inverse-variance-weighted estimate of the lensing potential $\left(\bar{\phi}_{L}\right)$ from two filtered CMB fields, i.e., $T T, T E, E E, E B$, and $T B$.

2. Remove a "MF" bias.

3. Normalize the estimate. In the $\phi$-estimator we use in this paper, the correct normalization of the estimate of $\phi$ for a given value of $\boldsymbol{L}$. is one over the total inversevariance weight. The best estimate of the lensing potential after normalization will be designated with a hat symbol, $\hat{\phi}_{L}$.

Using two CMB fields $\bar{X}$ and $\bar{Y}$ that have been filtered as described in Section 3.1, we estimate the inverse-varianceweighted lensing potential as follows:

$$
\bar{\phi}_{L}^{X Y}=\int d^{2} \ell W_{\ell, \ell-L}^{X Y} \bar{X}_{\ell} \bar{Y}_{\ell-L}^{*}
$$

where $W_{\ell, \ell-L}^{X Y}$ is a weight function as described below. Analogous to $\bar{X}$, we continue to use the over-bar notation to indicate that $\bar{\phi}_{L}$ is an inverse-variance-weighted field. This estimation technique amounts to taking a weighted sum of the covariance between $\bar{X}_{\ell}$ and $\bar{Y}_{\ell^{\prime}}$ at all pairs of angular wavenumbers $\ell$ and $\ell^{\prime}$ separated by $\ell-\ell^{\prime}=\boldsymbol{L}$. As indicated by Equation (5), modes $\ell$ in $\bar{X}$ and $\ell^{\prime}$ in $\bar{Y}$ have a covariance that is imprinted by modes in the lensing potential $\phi$ with angular wavenumber $\boldsymbol{L}$.

The weight function $W_{\ell, \ell-L}^{X Y}$ acts like a matched filter; in Equation (9) this weight is convolved in Fourier-space with the two filtered CMB fields to maximize the response to the lensing signal. We use the MV estimator for this analysis (Hu \& Okamoto 2002), in which this weight function is simply the leading-order coefficient on $\phi$ in Equation (5): $W_{\ell, \ell-L}^{X Y} \equiv W_{\ell, \ell-L}^{\phi^{X Y}}{ }^{38}$ In general, other weight functions can be used (for example, "bias-hardened" estimators defined in Namikawa et al. 2013).

This estimator is biased since it includes contributions that arise from the statistical anisotropy introduced by non-lensing sources such as the sky mask, in-homogeneous map noise, etc.

\footnotetext{
38 In contrast to $\mathrm{Hu} \&$ Okamoto (2002), who use the unlensed CMB spectrum in this definition, we use the lensed CMB spectrum following the treatment of Lewis et al. (2011).
}

These terms constitute a MF bias, defined as

$$
\bar{\phi}_{L}^{X Y, \mathrm{MF}}=\int d^{2} \ell W_{\ell, \ell-L}^{X Y}\left\langle\bar{X}_{\ell} \bar{Y}_{\ell-L}^{*}\right\rangle,
$$

where the average is taken over realizations of the CMB and noise. We calculate the MF contribution by averaging over estimates of $\phi$ from Monte-Carlo (MC) simulations with independent realizations of the CMB, lensing potential, and instrument noise. Since the simulated lensing signal is uncorrelated from simulation to simulation, it averages to zero in this calculation; the common signal that remains after averaging is the MF bias.

Finally, we need to normalize the response of the estimator to produce an unbiased estimate of $\phi$. This unbiased estimate will be designated with a hat symbol, $\hat{\phi}_{L}$. We calculate this normalization in a two-step process. We analytically calculate the normalization as a 2D Fourier-space object, then correct the analytic normalization using simulations.

Drawing on the intuition that the estimator is a weighted sum of off-diagonal elements in the covariance between $\bar{X}_{\ell}$ and $\bar{Y}_{\ell^{\prime}}$, the desired normalization is the reciprocal of the sum of these weights. To properly calculate the normalization, however, we must account for the map-filtering process described in Section 3.1. For the sky signal $X_{\ell}$ in our maps (specifically, $X_{\ell}$ as it appears in Equation (6)), the $C$-inverse filter can be described as an operator in two-dimensional Fourier-space that can mix power between $\ell$-modes. The $C$-inverse filter, however, can be approximated as a diagonal Fourier-space filter function of the form: $\left[C_{\ell}^{X X}+C_{\ell}^{N N}\right]^{-1} X_{\ell} \equiv \mathcal{F}_{\ell}^{X} X_{\ell}$, where $C_{\ell}^{X X}$ is the power spectrum of field $X_{\ell}$, and $C_{\ell}^{N N}$ is the power spectrum of the map noise (in which the beam $\left(F_{\ell}^{\text {beam }}\right)$ and filter-transfer function $\left(F_{\ell}^{\text {filt }}\right)$ have been divided out in Fourierspace; see Section 2 for more detail). We use this diagonal approximation for one thing only: to analytically estimate the normalization of the $\phi$-estimator.

Under this approximation, the normalization is given by

$$
\mathcal{R}_{L}^{X Y, \text { Analytic }}=\int d^{2} \ell W_{\ell, \ell-L}^{X Y} \times W_{\ell, \ell-L}^{\phi^{X Y}} \mathcal{F}_{\ell}^{X} \mathcal{F}_{\ell-L}^{Y} .
$$

Note that in the absence of filtering (i.e., $\mathcal{F}_{\ell}=1$ ), this equation is just the sum of the weights from Equation (9). The approximation $\mathcal{F}_{\ell}^{X}$ is not exact; we therefore calculate a multiplicative normalization correction $\mathcal{R}_{L}^{X Y, \mathrm{MC}}$ from simulations,

$$
\mathcal{R}_{L}^{X Y, \mathrm{MC}}=\frac{\left\langle\hat{\phi}_{L}^{I^{\prime}, X Y} \phi_{L}^{I *}\right\rangle}{\left\langle\phi_{L}^{I} \phi_{L}^{I *}\right\rangle},
$$

where for each simulated realization, $\phi_{L}^{I}$ is the input and

$$
\hat{\phi}_{L}^{I^{\prime}, X Y}=\frac{1}{\mathcal{R}_{L}^{X Y, \text { Analytic }}}\left(\bar{\phi}_{L}^{X Y}-\bar{\phi}_{L}^{X Y, \mathrm{MF}}\right)
$$

is the matching reconstruction, normalized by the analytic normalization. The reconstruction $\hat{\phi}_{L}^{I^{\prime}, X Y}$ is masked, the denominator is taken directly from the harmonic-space input maps, and the appropriate factor of $f_{\text {mask }}$ (see Equation (18)) is applied. The average is taken over 400 simulations. In principle (or the limit of many, many simulations), the correct normalization would be the product of $\mathcal{R}_{L}^{X Y \text {, Analytic }}$ with $\mathcal{R}_{L}^{X Y, \mathrm{MC}}$ in the 
2D $\boldsymbol{L}$-plane, however, each individual mode in $\mathcal{R}_{L}^{X Y, \mathrm{MC}}$ is still very noisy, making this method intractable. We expect this normalization to be isotropic, so we average $\mathcal{R}_{L}^{X Y, \mathrm{MC}}$ within annuli in $\boldsymbol{L}$-space to produce

$$
\mathcal{R}_{L}^{X Y, \mathrm{MC}}=\left\langle\mathcal{R}_{L}^{X Y, \mathrm{MC}}\right\rangle
$$

The normalization we use is the product of the analytically calculated normalization with the annulus-averaged $\mathrm{MC}$ correction:

$$
\mathcal{R}_{L}^{X Y} \equiv \mathcal{R}_{L}^{X Y, \text { Analytic }} \times \mathcal{R}_{L}^{X Y, \mathrm{MC}}
$$

We expect the MC correction to be relatively small, and indeed, we find that $\mathcal{R}_{L}^{X Y, \mathrm{MC}}$ is a $\leqslant 10 \%$ correction.

For each combination of fields $X$ and $Y$, our estimator of the lensing potential is defined as

$$
\hat{\phi}_{L}^{X Y}=\frac{1}{\mathcal{R}_{L}^{X Y}}\left(\bar{\phi}_{L}^{X Y}-\bar{\phi}_{L}^{X Y, \mathrm{MF}}\right) .
$$

We calculate all five temperature and polarization estimators $X Y \in[T T, T E, E E, E B, T B]$ (a quadratic estimate of $\phi$ from $B B$ has vanishing signal-to-noise in a cosmology with negligible gravitational wave perturbations ${ }^{39}$ Lewis \& Challinor 2006).

We now want to combine these estimates into one MV estimate of the lensing potential, denoted by $\hat{\phi}_{L}^{\mathrm{MV}}$. We form $\hat{\phi}_{L}^{\mathrm{MV}}$ from a weighted average of the five estimators, where the optimal weight $w_{L}^{X Y}$ for the MV estimator is $\mathcal{R}_{L}^{X Y}$. This can be understood as follows. In quadratic maximum likelihood estimators, $\mathcal{R}_{L}^{X Y}$ is the Fisher matrix for $\phi_{L}^{X Y}$; this means that the un-normalized estimate $\bar{\phi}-\bar{\phi}^{\mathrm{MF}}$ is the inverse-varianceweighted lens reconstruction. Thus we calculate the average of the inverse-variance-weighted estimators $\left(\bar{\phi}-\bar{\phi}^{\mathrm{MF}}\right)$, and divide by the sum of the normalizations. This is equivalent to calculating a weighted-sum of the $\hat{\phi}$ estimators, where the weight $w_{L}^{X Y}$ is the normalization, $\mathcal{R}_{L}^{X Y}$. For each $\boldsymbol{L}$ mode,

$$
\hat{\phi}_{L}^{\mathrm{MV}}=\frac{\sum_{X Y} w_{L}^{X Y} \hat{\phi}_{L}^{X Y}}{\sum_{X Y} w_{L}^{X Y}},
$$

where the sum is taken over $X Y \in[T T, T E, E T, E E$, $E B, B E, T B, B T]$. We additionally form a polarization-only (POL) estimate $\hat{\phi}_{L}^{\mathrm{POL}}$ from a weighted average of the $E E, E B$, and $B E$ estimators. Maps and power spectra of the lensing potential are calculated from both the MV and POL estimators.

\subsection{The Power Spectrum of $\phi$}

In this section, we present our method for estimating the power spectrum of the lensing potential. The steps are as follows, with a more detailed description given below.

\footnotetext{
39 This can be understood intuitively as follows. A cosmology with negligible gravitational wave perturbations has negligible primordial $B$-mode power. The main contribution of $B$-mode power will come from $E$-modes that have been lensed. This means that when the $B$-mode power is expanded as Taylor-series in the lensing potential $\phi$, the leading-order term will be first-order and higher in $\phi$. The quadratic $B B$ estimator is constructed from two copies of the $B$-mode field; therefore, the leading term will be second-order in $\phi$ and will thus be negligible.
}

1. Calculate a cross-spectrum $\left(C_{L}^{\hat{\phi}^{U V} \hat{\phi}^{X Y}}\right)$ from two estimates of $\phi, \hat{\phi}_{L}^{U V}$ and $\hat{\phi}_{L}^{X Y}$.

2. Calculate and subtract noise-bias terms $\left(\left.\Delta C_{L}^{\phi^{U V} \phi^{X Y}}\right|_{\mathrm{RDN} 0}\right.$ and $\left.\Delta C_{L}^{\phi^{U V} \phi^{X Y}}\right|_{\mathrm{N} 1}$ ). The de-biased spectrum for this combination of CMB fields is denoted as $\hat{C}_{L}^{\phi^{U V} \phi^{X Y}}$, with a hat symbol to indicate that this is our best estimate.

3. Average this spectrum into bins in $L$. The binned spectrum we report is denoted as $\hat{C}_{L_{b}}^{\phi^{U V} \phi^{X Y}}$.

4. Finally, calculate the amplitude of $\hat{C}_{L}^{\phi^{U V} \phi^{X Y}}$ relative to a fiducial spectrum. Note, the amplitude is calculated directly from the 2D $\boldsymbol{L}$-plane, rather than from the binned spectrum.

The power spectrum from two estimates $\hat{\phi}^{U V}$ and $\hat{\phi}^{X Y}$ is

$$
C_{L}^{\hat{\phi}^{U V} \hat{\phi}^{X Y}} \equiv f_{\text {mask }}^{-1}\left\langle\hat{\phi}_{L}^{U V} \hat{\phi}^{*}{ }_{L}^{X Y}\right\rangle,
$$

where $f_{\text {mask }}$ is the average value of the fourth power of the apodization and point-source mask. In the following discussion, we will drop the $U V, X Y$ superscripts $\left(C_{L}^{\phi \phi}\right)$ unless they are needed for clarity. Note that in this power spectrum calculation, the MF correction $\phi^{\mathrm{MF}}$ is estimated from two independent sets of simulations for each of the two $\hat{\phi}$ estimates. We must do this because our estimates of the MF are noisy, owing to a finite number of simulations. By using separate estimates of the $\mathrm{MF}$, we eliminate correlations between the two $\hat{\phi}$ estimates resulting from this noisy subtraction.

While this estimate of the $C_{L}^{\phi \phi}$ power spectrum has minimal variance, it suffers from additive biases which we must subtract. We now describe these additive bias terms. The estimates of $\phi$ are quadratic, i.e., 2-point functions of the CMB fields, thus the cross-spectrum $C_{L}^{\hat{\phi} \hat{\phi}}$ between estimates of $\phi$ probes the 4-point function (trispectrum) of the CMB. The CMB trispectrum includes contributions from disconnected and connected pieces, only some of which contain information about $C_{L}^{\phi \phi}$. The remaining correlations show up as "noise bias" terms in $C_{L}^{\hat{\phi} \hat{\phi}}$ (Kesden et al. 2003). At a given order, the disconnected pieces arise from lower-order correlations, while the connected pieces are new at each order. In the case of the CMB trispectrum, the 2-point (Gaussian) correlations in the $\mathrm{CMB}$ give rise to the disconnected pieces, while the higher-order (non-Gaussian) correlations introduced by lensing give rise to the connected pieces. We model the full cross-spectrum, including bias terms, as:

$$
C_{L}^{\hat{\phi} \hat{\phi}}=\hat{C}_{L}^{\phi \phi}+\left.\Delta C_{L}^{\phi \phi}\right|_{\mathrm{N} 0}+\left.\Delta C_{L}^{\phi \phi}\right|_{\mathrm{N} 1}+\left.\Delta C_{L}^{\phi \phi}\right|_{\mathrm{MC}}
$$

where $\hat{C}_{L}^{\phi \phi}$ is the term we want to calculate, $\left.\Delta C_{L}^{\phi \phi}\right|_{\text {NO }}$ and $\left.\Delta C_{L}^{\phi \phi}\right|_{\mathrm{N} 1}$ are the disconnected and connected pieces of the trispectrum, respectively, and $\left.\Delta C_{L}^{\phi \phi}\right|_{\mathrm{MC}}$ encapsulates any remaining bias terms. We discuss each of these terms below, and show them in Figure 1.

The first bias term $\left.\Delta C_{L}^{\phi \phi}\right|_{\mathrm{N} 0}$ arises from disconnected contributions to the trispectrum. This term has no dependence 


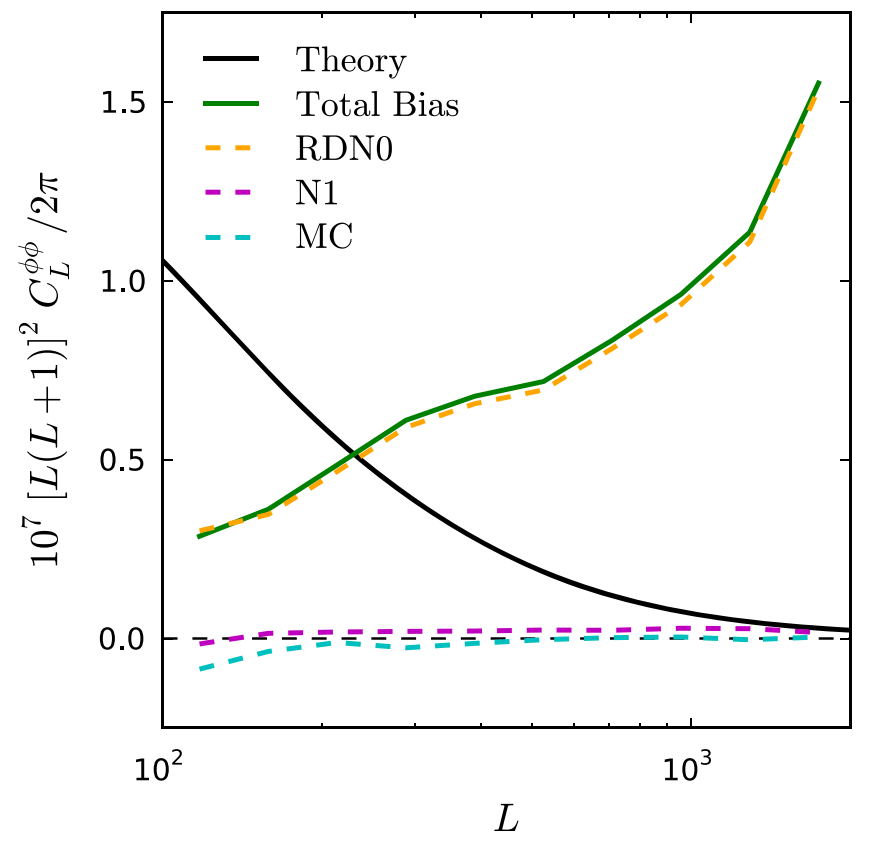

Figure 1. Noise-bias levels for this $\phi$ reconstruction. The theoretical $\Lambda$ CDM lensing potential power spectrum is shown in black, and the individual bias terms defined in Equation (19) are shown individually. The realizationdependent NO (RDN0) term (dashed orange line) is used to correct the data for the Gaussian noise bias (see Equation (21)). The N1 bias (dashed purple line) arises from connected contributions to the CMB trispectrum (see Equation (22)). The sum of these two terms is the total bias (solid green line) that we subtract. The residual Monte-Carlo (MC) bias term (dashed cyan line) from Equation (19) is calculated as the difference between the mean of de-biased lensed simulation spectra and the input spectrum to the simulations. The total bias shows the reconstruction-noise power in the reconstructed $\phi$ maps; thus this reconstruction measures $\phi$ modes with a signal-to-noise ratio greater than 1 (alternatively, the measurement is $\phi$-sample-variance limited) for $100<L \lesssim 250$.

on $\phi$ and arises from Gaussian correlations in the CMB fields, foregrounds, and noise; this term is called "NO" because it is zeroth-order in $C_{L}^{\phi \phi}$. We estimate the N0 contribution from simulations in the following way: we create two sets of simulations, $\mathrm{MC}$ and $\mathrm{MC}^{\prime}$, with different realizations of the $\mathrm{CMB}$ and $\phi$ (including foregrounds and noise). The prescription for calculating $\mathrm{N} 0$ from the $\mathrm{MC}$ and $\mathrm{MC}^{\prime}$ simulations can be written as the sum of two terms:

$$
\begin{aligned}
\left.\Delta C_{L}^{\phi \phi}\right|_{\mathrm{N} 0}= & \left\langle+C_{L}^{\hat{\phi} \hat{\phi}}\left[\bar{U}_{\mathrm{MC}}, \bar{V}_{\mathrm{MC}^{\prime}}, \bar{X}_{\mathrm{MC}}, \bar{Y}_{\mathrm{MC}^{\prime}}\right]\right. \\
& \left.+C_{L}^{\hat{\phi} \hat{\phi}}\left[\bar{U}_{\mathrm{MC}}, \bar{V}_{\mathrm{MC}^{\prime}}, \bar{X}_{\mathrm{MC}^{\prime}}, \bar{Y}_{\mathrm{MC}}\right]\right\rangle_{\mathrm{MC} \mathrm{MC}^{\prime}}
\end{aligned}
$$

where we have re-written $C_{L}^{\hat{\phi}^{U V} \hat{\phi}^{X Y}}$ to explicitly show the dependence on the four input fields $U, V, X, Y$ : $C_{L}^{\hat{\phi} \hat{\phi}}[\bar{U}, \bar{V}, \bar{X}, \bar{Y}] \equiv C_{L}^{\hat{\phi}^{U V}} \hat{\phi}^{X Y}$.

This estimate of the N0 bias for the data will be imperfect because the power spectrum used in the simulations will be different from that of the data. We can reduce our sensitivity to this difference by using the data itself, a correction called the "realization-dependent NO" (RDNO) bias (Namikawa et al. 2013). We calculate the RDN0 bias by replacing one of the CMB fields in the estimate of N0 with the data itself, then combining it with the NO estimate above:

$$
\begin{aligned}
\left.\Delta C_{L}^{\phi \phi}\right|_{\mathrm{RDN} 0} & \\
= & \left\langle+C_{L}^{\hat{\phi} \hat{\phi}}\left[\bar{U}_{\mathrm{d}}, \bar{V}_{\mathrm{MC}}, \bar{X}_{\mathrm{d}}, \bar{Y}_{\mathrm{MC}}\right]\right. \\
& +C_{L}^{\hat{\phi} \hat{\phi}}\left[\bar{U}_{\mathrm{MC}}, \bar{V}_{\mathrm{d}}, \bar{X}_{\mathrm{d}}, \bar{Y}_{\mathrm{MC}}\right] \\
& +C_{L}^{\hat{\phi} \hat{\phi}}\left[\bar{U}_{\mathrm{d}}, \bar{V}_{\mathrm{MC}}, \bar{X}_{\mathrm{MC}}, \bar{Y}_{\mathrm{d}}\right] \\
& +C_{L}^{\hat{\phi} \hat{\phi}}\left[\bar{U}_{\mathrm{MC}}, \bar{V}_{\mathrm{d}}, \bar{X}_{\mathrm{MC}}, \bar{Y}_{\mathrm{d}}\right] \\
& -C_{L}^{\hat{\phi} \hat{\phi}}\left[\bar{U}_{\mathrm{MC}}, \bar{V}_{\mathrm{MC}}, \bar{X}_{\mathrm{MC}}, \bar{Y}_{\mathrm{MC}}\right] \\
& \left.-C_{L}^{\hat{\phi} \hat{\phi}}\left[\bar{U}_{\mathrm{MC}}, \bar{V}_{\mathrm{MC}}, \bar{X}_{\mathrm{MC}^{\prime}}, \bar{Y}_{\mathrm{MC}}\right]\right\rangle_{\mathrm{MC}, \mathrm{MC}^{\prime}},
\end{aligned}
$$

where the $d$ subscript indicates a CMB field from the data. We subtract this RDN0 estimate from the data spectrum.

The second bias term $\left.\Delta C_{L}^{\phi \phi}\right|_{\mathrm{N} 1}$ arises from connected contributions to the trispectrum and depends linearly on $C_{L}^{\phi \phi}$ (Kesden et al. 2003); this term is called "N1" because it is firstorder in $C_{L}^{\phi \phi}$. We estimate this term by creating two sets of simulations, $\mathrm{MC}$ and $\mathrm{MC}^{\prime}$, in which each simulated pair has the same realization of $\phi$, but different realizations of the unlensed CMB; see Section 4 for more detail. The N1 term is then ${ }^{40}$

$$
\begin{aligned}
\left.\Delta C_{L}^{\phi \phi}\right|_{\mathrm{N} 1}= & \left\langle+C_{L}^{\hat{\phi} \hat{\phi}}\left[\bar{U}_{\phi^{1}, \mathrm{MC}}, \bar{V}_{\phi^{1}, \mathrm{MC}}, \bar{X}_{\phi^{1}, \mathrm{MC}}, \bar{Y}_{\phi^{1}, \mathrm{MC}}\right]\right. \\
& +C_{L}^{\hat{\phi} \hat{\phi}}\left[\bar{U}_{\phi^{1}, \mathrm{MC}}, \bar{V}_{\phi^{1}, \mathrm{MC}}, \bar{X}_{\phi^{1}, \mathrm{MC}}, \bar{Y}_{\phi^{1}, \mathrm{MC}}\right] \\
& \left.-\left.\Delta C_{L}^{\phi \phi}\right|_{\mathrm{N} 0}\right\rangle_{\mathrm{MC}, \mathrm{MC}^{\prime}} .
\end{aligned}
$$

The final term $\left.\Delta C_{L}^{\phi \phi}\right|_{\mathrm{MC}}$ encapsulates any corrections that have not been accounted for as yet. We calculate this term as the difference of the simulations from the input spectrum:

$$
\left.\Delta C_{L}^{\phi \phi}\right|_{\mathrm{MC}}=C_{L}^{\hat{\phi} \hat{\phi}} \mathrm{MC}-\left.\Delta C_{L}^{\phi \phi}\right|_{\mathrm{N} 0}-\left.\Delta C_{L}^{\phi \phi}\right|_{\mathrm{N} 1} .
$$

We find that $\left.\Delta C_{L}^{\phi \phi}\right|_{\mathrm{MC}}$ is small and do not subtract it in our final estimate; see Section 5 for further discussion.

Thus our final measured power spectrum may be written as

$$
\hat{C}_{L}^{\phi \phi}=C_{L}^{\hat{\phi} \hat{\phi}}-\left.\Delta C_{L}^{\phi \phi}\right|_{\text {RDN0 }}-\left.\Delta C_{L}^{\phi \phi}\right|_{\mathrm{N} 1} .
$$

Now that we have the best estimate of our lensing power spectrum $\hat{C}_{L}^{\phi \phi}$, we would like to average this spectrum into bins in $L$. The power in each bin is referred to as a "bandpower." Because $\hat{C}_{L}^{\phi \phi}$ is a two-dimensional quantity with varying signal-to-noise across the $2 \mathrm{D} \boldsymbol{L}$-plane, a naive binning operation of simply averaging $\hat{C}_{L}^{\phi \phi}$ in each bin is sub-optimal. Instead, we calculate the weighted average of $\hat{C}_{L}^{\phi \phi}$ within each bin:

$$
C_{b}^{\phi^{U V} \phi^{X Y}} \equiv \frac{\sum_{L \in b} w_{L}^{U V X Y} \hat{C}_{L}^{\phi^{U V} \phi^{X Y}}}{\sum_{L \in b} w_{L}^{U V X Y}} .
$$

The choice of the weight function $w_{L}^{U V X Y}$ follows from the same reasoning used to define the weights in Equation (17).

\footnotetext{
${ }^{40}$ Note, Gaussian foreground power is not included in the simulations for N1.
} 
Specifically, in the quadratic maximum likelihood estimator that we are using, $\mathcal{R}_{L}^{X Y}$ is the Fisher matrix for $\phi_{L}^{X Y}$; therefore the inverse-variance weight for $\hat{C}_{L}^{\phi \phi}$ is the product of the normalization of the two estimates $\phi^{U V}$ and $\phi^{X Y}$ :

$$
w_{L}^{U V X Y}=\mathcal{R}_{L}^{U V} \mathcal{R}_{L}^{X Y} .
$$

This weight scheme is analytically optimal in two cases: either for an estimator of the auto-spectrum where $U=V=X=Y$ or for a complete set of cross-spectra between different estimators (e.g., the MV and POL estimators).

We calculate the amplitude $A_{b}$ of our data spectrum relative to a theory spectrum within a bin $b$,

$$
A_{b}^{U V X Y} \equiv \frac{C_{b}^{\phi^{U V} \phi^{X Y}}}{C_{b}^{\phi^{U V} \phi^{X Y}, \text { theory }}},
$$

where the binned theory spectrum is

$$
C_{b}^{\phi^{U V} \phi^{X Y}, \text { theory }} \equiv \frac{\sum_{L \in b} w_{L}^{U V X Y} C_{L}^{\phi \phi, \text { theory }}}{\sum_{L \in b} w_{L}^{U V X Y}} .
$$

We report bandpowers $\hat{C}_{L_{b}}^{\phi \phi}$ as the data-amplitude $A_{b}$ multiplied by the theoretical spectrum $C_{L_{b}}^{\phi \phi, \text { theory }}$ evaluated at the bin center $L_{b}$,

$$
\hat{C}_{L_{b}}^{\phi \phi} \equiv A_{b} C_{L_{b}}^{\phi \phi, \text { theory }} .
$$

Finally, we calculate two overall amplitudes of $\hat{C}_{L}^{\phi \phi}$ relative to a theory spectrum: $A_{\mathrm{MV}}$ for the MV spectrum and $A_{\mathrm{POL}}$ for the polarization-only spectrum. These amplitudes are calculated using Equation (27), where the "bin" is taken to the full range $100<L<2000$. The reference theory spectrum $C_{L}^{\phi \phi}$,theory is taken to be the PLANCK+LENS+WP+HIGHL spectrum. We additionally report the spectrum bandpowers as defined in Equation (29), which contain the information about the shape of our measured spectrum.

\section{SIMULATIONS}

The lensing analysis presented here relies heavily on accurate simulations. We use the spectra from the PLANCK +LENS+WP+HIGHL model as our fiducial cosmological model for simulations. We create simulations as follows.

First, we create realizations of spherical-harmonic coefficients $\left(a_{l m}\right)$ for the CMB fields $T, E$, and $B$ (including the proper correlations between the fields), as well as the lensing potential $\phi$. We simulate modes with $\ell<6000$. We evaluate the spherical harmonic transform ${ }^{41}$ of the $a_{l m}$ coefficients on a grid with an equidistant cylindrical projection (ECP) (Lewis 2005). The lensing operation is applied by distorting the unlensed fields using the deflection map derived from $\phi$. These distorted maps are interpolated back onto a fixed ECP grid, creating lensed $\mathrm{CMB}$ skies with full non-Gaussian information.

These maps are then mock-observed with the map-making pipeline. This involves creating simulated TOD for each bolometer, filtering the TOD, accumulating the TOD into flatsky maps using the SPT pointing information, and coadding

\footnotetext{
${ }^{41}$ The spherical harmonic transform is performed with routines from the HEALPIX library (Górski et al. 2005).
}

different detectors based on the individual detector weights. In effect, the resulting maps are what SPT would have seen if these simulated skies had been the true CMB sky, in the absence of noise and foregrounds.

The mock-observed maps are transformed back into Fourier space, where Gaussian foreground power is added. We use the foreground model from Story et al. (2013), with the following components: $D_{3000}^{\mathrm{PS}}=10 \mu \mathrm{K}^{2}$ is the power from Poissondistributed point-sources that scales as $D^{\mathrm{PS}} \propto \ell^{2}$, $D_{3000}^{\mathrm{CL}}=5 \mu \mathrm{K}^{2}$ is the power from clustered CIB sources that scales as $D^{\mathrm{CL}} \propto \ell^{0.8}, D_{3000}^{\mathrm{SZ}}=5 \mu \mathrm{K}^{2}$ is the amplitude of the tSZ power spectrum that we use to scale the thermal SZ template taken from Shaw et al. (2010). The three coefficients here are given at $\ell=3000$. See Section 6.1 of Story et al. (2013) for details. The Fourier-space maps are multiplied by the Fourier-space SPT beam $F_{\ell}^{\text {beam }}$.

We then add realizations of noise to the simulations. These realizations are estimated directly from the data. We take all observations and divide them into two halves, and subtract the coadd of one half from the coadd of the other half. We create many realizations of the noise by choosing random sets of halves. This method of calculating the noise variance gives an unbiased but potentially noisy estimate; however, given the number of independent observations used in this work, the noise on the variance estimate is expected to be negligible.

We make three sets of simulations:

1. Set "A": 500 lensed simulations.

2. Set "B": 100 lensed simulations with different realizations of the CMB but the same realizations of $\phi$ as the first 100 simulations in Set A.

3. Set "C": 500 unlensed simulations.

These simulations are used as follows. The simulations in set "A" are used to calculate the MF (see Section 3.2), the N0 bias term (see Section 3.3), and the statistical uncertainty (see Section 5). Specifically, the first 100 simulations are used to calculate the MF; in each cross-spectrum we use the first 50 simulations to estimate the MF of the first $\phi$ and the second 50 simulations to estimate the MF of the second $\phi$. The remaining 400 simulations are used to calculate the statistical uncertainty on the lensing spectrum and amplitude. The entire set is used to calculate $\left.\Delta C_{L}^{\phi \phi}\right|_{\mathrm{RDN} 0}$.

The simulations in set "B" are used to calculate $\left.\Delta C_{L}^{\phi \phi}\right|_{\mathrm{N} 1}$, as described in Equation (22). Specifically, the simulations labeled MC are comprised of 50 simulations from set "A," while the simulations labeled $\mathrm{MC}^{\prime}$ come from 50 matching simulations from set "B."

Finally, the unlensed simulations that comprise set "C" are used for two purposes. First, we check that no spurious lensing in our pipeline is detected by measuring the amplitude of the reconstructed lensing potential from these unlensed simulations. The average amplitude and variance of these simulations is $A_{\text {unl }}=-0.024 \pm 0.065$, thus passing this test. Second, we quantify how significantly we reject the no-lensing hypothesis by comparing the lensing amplitude measured in the data to the variance of these unlensed simulations. The first 100 simulations are used to calculate the MF, and the remaining 400 simulations are used to calculate the statistical uncertainty of unlensed skies. 
Table 1

Systematic Uncertainties

\begin{tabular}{lll}
\hline \hline Type & $\Delta A_{\mathrm{MV}}$ & $\Delta A_{\mathrm{POL}}$ \\
\hline$\Delta A_{\text {Tcal }}$ & 0.052 & 0.052 \\
$\Delta A_{\text {Pcal }}$ & 0.030 & 0.067 \\
$\Delta A_{\text {crosstalk }}$ & 0.05 & 0.06 \\
$\Delta A_{\text {amp }}$ & 0.029 & 0.039 \\
\hline$\Delta A_{\text {tot }}$ & & 0.11 \\
\hline
\end{tabular}

Note. The contributions to the systematic uncertainty budget. The quadrature sum of the systematic uncertainty terms gives the total systematic uncertainty, $\Delta A_{\text {tot }}$

In Figure 7, the distribution of lensing amplitudes from these lensed simulations is shown in green, and the distribution for unlensed simulations is shown in red.

\section{UNCERTAINTY BUDGET}

The uncertainties on the lensing spectrum and amplitude are comprised of variance (noise and sample), calibration and beam error, and other systematic errors. The variance component is calculated as the covariance between the power spectra of 400 lensed simulations. These simulations use independent realizations of the unlensed CMB, lensing potential, and instrumental noise, thus this procedure naturally accounts for both instrumental noise and sample variance.

We also account for sources of systematic uncertainty. The resulting uncertainty on the lensing amplitude is calculated below and shown in Table 1.

\subsection{Beam and Absolute Calibration}

The estimation of the beam and absolute calibration contributes additional systematic uncertainty. The fractional uncertainties on the beam measurements are less than $1 \%$ over the multipoles used in this analysis, and the resulting uncertainty on the lensing amplitude is negligible. The $1 \sigma$ uncertainly on the absolute calibration in temperature is $\delta_{\text {Tcal }}=1.3 \%(\mathrm{C} 14)$. This will propagate into an uncertainty on the spectrum amplitude of $\Delta A_{\text {Tcal }}=A\left(1+\delta_{\text {Tcal }}\right)^{4}$. We thus incorporate a systematic uncertainty on the amplitude from calibration and beams of $\Delta A_{\mathrm{Tcal}}=0.052$.

\subsection{Polarization Calibration}

Similarly, an error in the polarization calibration will propagate to an uncertainty on the spectrum amplitude of polarized estimators. We estimate the $1 \sigma$ uncertainty on the polarization calibration to be $\delta_{\text {Pcal }}=1.7 \%(\mathrm{C} 14)$. We incorporate a systematic uncertainty on the POL amplitude of $\Delta A_{\mathrm{POL}}^{\mathrm{Pcal}}=0.067$. We estimate the resulting systematic uncertainty in the MV amplitude by calculating the relative change in the analytic normalization $\mathcal{R}_{L}^{\mathrm{POL} \text {,Analytic }}$ resulting from a $1 \sigma$ shift in $P_{\text {cal }}$. We find an uncertainty in the $\mathrm{MV}$ amplitude of $\Delta A_{\mathrm{MV}}^{\mathrm{Pcal}}=0.030$.

\subsection{Temperature-to-polarization Leakage}

Mis-estimating the temperature power leaked into $Q$ and $U$ maps would bias the lensing measurement. We recalculate the MV lensing amplitude without correcting for the leakage. The amplitude changes by less than $0.07 \sigma$; this source of uncertainty is negligible.

\subsection{Electrical Crosstalk between Detectors}

There is some low-level electrical crosstalk between detectors. The main effect of crosstalk is to introduce a difference between the temperature and polarization instrumental beams, resulting in a small multiplicative bias in the polarization measurements; see $\mathrm{C} 14$ for a detailed discussion. The effect of crosstalk on this lensing analysis was investigated using the simulation pipeline described in Section 4 and adding the effect of crosstalk to the simulated timestreams. We find that crosstalk introduces a small bias in the lensing amplitude, which we estimate to be a $5 \%(6 \%)$ in the MV (POL) amplitude; we account for this by adding a contribution to our systematics uncertainty budget of $\Delta A_{\mathrm{MV}}^{\text {crosstalk }}=0.05$ and $\Delta A_{\mathrm{POL}}^{\text {crosstalk }}=0.06$. The bias in the data from crosstalk is expected to be smaller than this estimate, because the data uses an RDN0 bias subtraction that reduces the effect of a mismatch between the power in the simulations relative to the data itself. In contrast, these simulations use a normal N0 bias subtraction, rather than recalculating an RDN0 bias for each simulation. We keep this over-estimate of the crosstalk-induced bias and include this term in our systematic uncertainty budget.

\subsection{Foregrounds}

Foreground emission from extra-galactic sources and galactic dust contributes both Gaussian power and nonGaussian signal to CMB observations which, if not accounted for, will bias lensing reconstruction measurements. The Gaussian power component contributes to the N0 bias, which we subtract using the simulations described in Section 4 and procedure described in Section 3.3.

The non-Gaussian mode-coupling from foreground emission has been studied in detail in (van Engelen et al. 2014a, hereafter V14), and Osborne et al. (2014). The work in V14 is particularly relevant to our analysis. They studied a comprehensive list of potential biases to the temperature lensing reconstruction that arise from foregrounds; they considered Poisson-distributed galaxies, CIB emission from clustered galaxies, tSZ signal from galaxy clusters, galaxy-lensing correlations, and tSZ-lensing correlations. For the point-source and cluster masking thresholds used in our analysis, the bias to the lensing spectrum never exceeds a few percent. van Engelen et al. (2012) also tested diffuse Galactic cirrus emission and found that the bias was less than $2 \%$ in all $L$-bins.

The contribution to the NO bias from polarized foregrounds is negligible at the sensitivity level of this analysis since the polarized power from point sources is too low to be detected significantly in the $E E$ and $T E$ polarization spectra in C14, even with the significantly higher flux cut of $50 \mathrm{mJy}$ in that work. The non-Gaussian signature of polarized foregrounds is expected to be negligible as well: the polarization fraction of foreground emission is expected to be lower than the polarization fraction of the $\mathrm{CMB}$, implying that the ratio of non-Gaussian foreground signal to $\mathrm{CMB}$ lensing signal will be lower in polarization than temperature, and the non-Gaussian contribution in temperature has already been shown to be negligible for this analysis.

Finally, we consider whether emission from polarized Galactic dust could contaminate the polarized lensing 
estimators. The Gaussian power in $B$ modes from Galactic dust within the BICEP2 field was estimated in a joint analysis of BICEP2 and Planck data (BICEP2/Keck \& Planck Collaborations et al. 2015); because the BICEP2 field contains the SPTpol deep field, this analysis provides a good estimate of Galactic dust in the SPTpol data. The best-fit model from the joint analysis is $D^{\text {dust }}(\ell)=D_{\ell=80}^{\text {dust }} \times(\ell / 80)^{-0.42}$, where $D_{\ell=80}^{\text {dust }}=0.0118 \mu \mathrm{K}$ arcmin. Using this model, the dust power in this field is $\sim 10 \%$ of the lensed $B$-mode power and $\sim 1 \%$ of the $B$-mode noise power (as calculated in Keisler et al. 2015) at the lowest multipoles used in the SPTpol lensing analysis $(\ell>450)$, and the dust power drops rapidly with increasing $\ell$. Thus we conclude that the Gaussian power from Galactic dust in our polarized maps has a negligible contribution to both our observed signal and variance. Significant non-Gaussian contributions from any foreground sources, including Galactic dust, should cause a failure of the curl test since foregrounds should contain both gradient and curl modes. Both the MV and POL estimators pass the curl test, thus we conclude that Galactic dust does not contribute significantly to the observed lensing signal in either temperature or polarization.

We conclude that given the level of precision of this analysis and our level of source masking, the RDN0 bias correction sufficiently accounts for the Gaussian power from unpolarized foregrounds, and that the Gaussian power from polarized foregrounds can be neglected in the RDNO term. Furthermore, we conclude that the biases due to the non-Gaussian contribution from polarized and unpolarized foregrounds are negligible at the level of statistical precision of the current analysis.

\subsection{Normalization Calculation}

We find that the mean MV (POL) amplitude of our lensed simulations is $3 \%(4.5 \%)$ below unity; this can be seen as a shift in the histogram of lensed simulations in Figure 7. This bias could be an additive term (e.g., the $\left.\Delta C_{L}^{\phi \phi}\right|_{\mathrm{MC}}$ term from Equation (19)) or a multiplicative term (e.g., misestimation of the power-spectrum normalization). We treat this potential bias as a systematic uncertainty in our analysis of $\Delta A_{\mathrm{MV}}^{\mathrm{amp}}=+0.029$ and $\Delta A_{\mathrm{POL}}^{\mathrm{amp}}=+0.039$ on the $\mathrm{MV}$ and POL amplitudes, respectively.

\subsection{Total Uncertainty}

The four sources of significant systematic error described above are added in quadrature to calculate our final systematic uncertainty on the lensing amplitude: $\Delta A_{\mathrm{MV}}= \pm 0.08$ and $\Delta A_{\mathrm{POL}}= \pm 0.11$ on the amplitudes of the $\mathrm{MV}$ and $\mathrm{POL}$ spectra, respectively. Thus our uncertainty on the amplitude of the MV lensing power spectrum is $\Delta A_{\mathrm{MV}}= \pm 0.14$ (Stat.) \pm 0.08 (Sys.), and our uncertainty on the amplitude of the POL lensing power spectrum is $\Delta A_{\mathrm{POL}}= \pm 0.24$ (Stat.) \pm 0.11 (Sys.).

Finally, we calculate a total uncertainty by adding the statistical and systematic uncertainties in quadrature: $\Delta A_{\mathrm{MV}}^{\mathrm{tot}}= \pm 0.16$ and $\Delta A_{\mathrm{POL}}^{\text {tot }}= \pm 0.26$

\section{RESULTS}

We present three main results in this section: the MV map of the lensing convergence field, $\kappa(\hat{\boldsymbol{n}})$, the MV and POL estimates of the binned lensing potential power spectrum, and the amplitudes of these two spectra relative to the PLANCK+LENS $+\mathrm{WP}+$ HIGHL model.

\subsection{Lensing Potential Map}

We show measured lensing convergence maps ${ }^{42} \kappa(\hat{\boldsymbol{n}})$ in Figure 2. These $\kappa$-maps are the real-space equivalent of $(1 / 2) L(L+1) \hat{\phi}_{L}$, where $\hat{\phi}_{L}$ is defined in Equation (16). We show the MV map, as well as four of the five individual estimators. The similarities between the different estimators are visually apparent.

We measure lensing modes with a signal-to-noise ratio greater than one for modes between $100<L<250$; thus the large-scale features that are visible by eye are real overdensities and under-densities in the projected mass distribution in the universe. This is the highest signal-to-noise lensing map from the CMB to date.

\subsection{Lensing Potential Power Spectrum}

Here we present the bandpowers from our estimate of the lensing potential power spectrum. We report the bandpowers between $100<L<2000$. The lower boundary is set at 100 to maximize the signal while keeping the MC bias sub-dominant to other systematic uncertainties; the MC bias increases as this lower boundary is reduced. The upper boundary is deep in the noise-dominated region of the spectrum; we find that the statistical uncertainty on the lensing spectrum amplitude is roughly constant for any boundary value above $L \gtrsim 1000$ and $L \gtrsim 800$ for the MV and POL estimators, respectively. We calculate the cross-spectrum from each pair of estimators $\left[\hat{\phi}_{L}^{U V}, \hat{\phi}_{L}^{X Y}\right]$, as well as two $\mathrm{MV}$ estimates, $\hat{C}_{L_{b}}^{\mathrm{MV}}$ from the combination of all estimators, and $\hat{C}_{L_{b}}^{\mathrm{POL}}$ from the polarizationonly estimators $E E, E B$, and $B E$. The cross-spectra between each pair of these lensing potential estimators is shown in Figure 4.

The MV spectrum $\hat{C}_{L_{b}}^{\mathrm{MV}}$ and POL spectrum $\hat{C}_{L_{b}}^{\mathrm{POL}}$ are shown in Figure 5. The bandpowers for the MV spectrum are presented in Table 2. We measure the amplitude of the spectrum using Equation (27) relative to the PLANCK+LENS+WP + HIGHL model. We find an amplitude of $A_{\mathrm{MV}}=0.92 \pm 0.14$ (Stat.) \pm 0.08 (Sys.) for the combined spectrum and $A_{\mathrm{POL}}=0.92 \pm 0.24$ (Stat.) \pm 0.11 (Sys.) for the polarization-only spectrum. Thus we measure the amplitude of the lensing potential power spectrum using solely polarization estimators with a precision of $26 \%$; including systematic errors, this becomes a precision of $29 \%$. Similarly, the precision of the MV measurement of the amplitude is $15 \%$; including systematic errors, this becomes a precision of $18 \%$.

We quantify how significantly we reject the null hypothesis of no lensing by comparing the data to unlensed simulations. In 400 unlensed simulations, none have a lensing amplitude as large as that in the data in either the MV or POL estimator. To estimate statistical significance, we fit a Gaussian to the 400 unlensed simulations, yielding a $14 \sigma$ and $5.9 \sigma$ rejection of the null hypothesis for the MV and POL estimators, respectively.

Figure 7 demonstrates the difference between these constraints. We rule out the no-lensing hypothesis much more

\footnotetext{
42 The map is presented as $\kappa$ because this corresponds visually to the density of the integrated matter field along the line of sight: $\kappa>0$ corresponds to an over-density that "stretches" the observed CMB pattern, while $\kappa<0$ corresponds to an under-density that "contracts" the observed CMB pattern.
} 

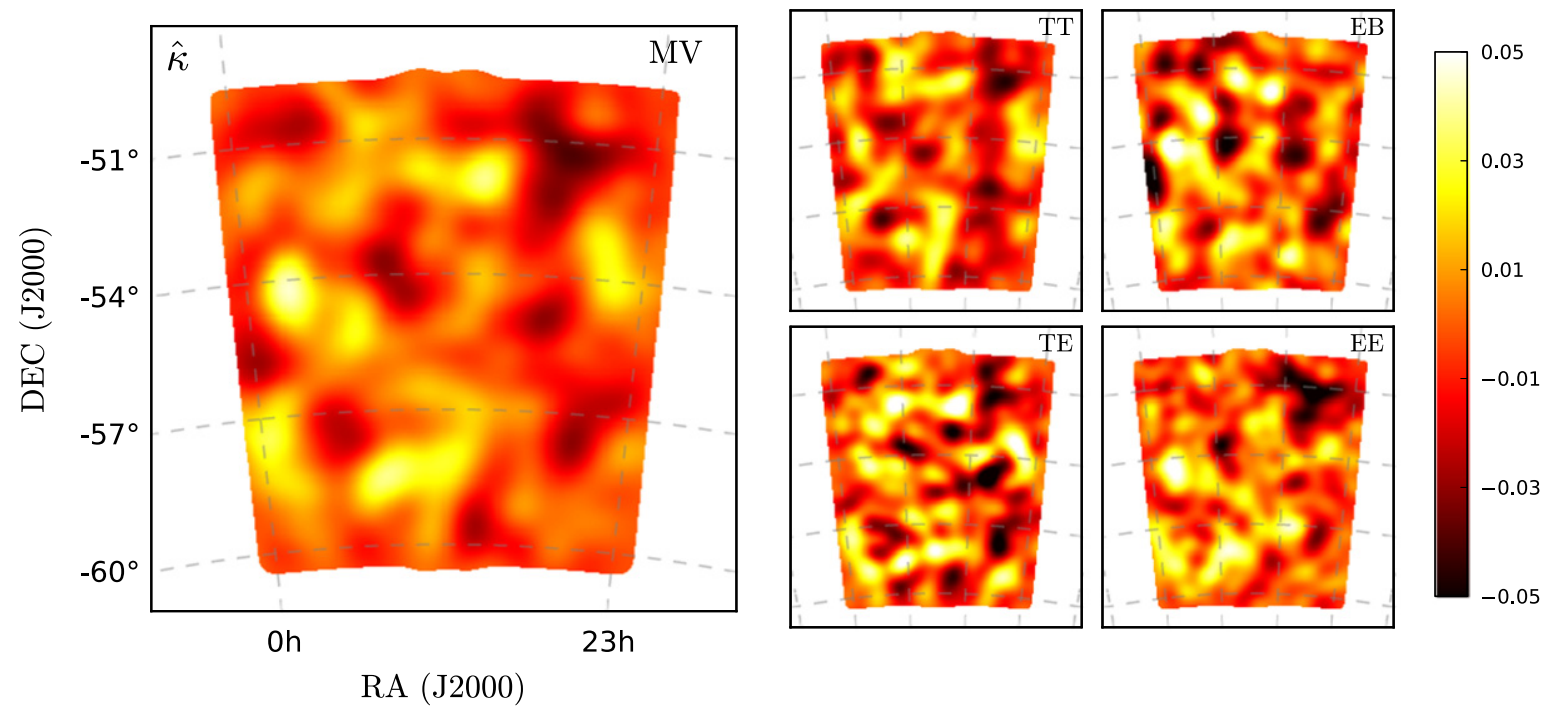

RA (J2000)

Figure 2. Lensing $\kappa$ maps reconstructed from the SPTpol $100 \mathrm{deg}^{2}$ deep-field data, smoothed with a $1^{\circ}$ Gaussian beam. The colorbar on the far right shows the color scale, which has been fixed for all $\kappa$ maps in Figures 2 and 3. Left: the $\kappa$-map for our MV lensing estimator, which combines all temperature and polarization information. Right: individual $\kappa$ estimates from the $T T, E B, T E$, and $E E$ estimators, with the same color scale.
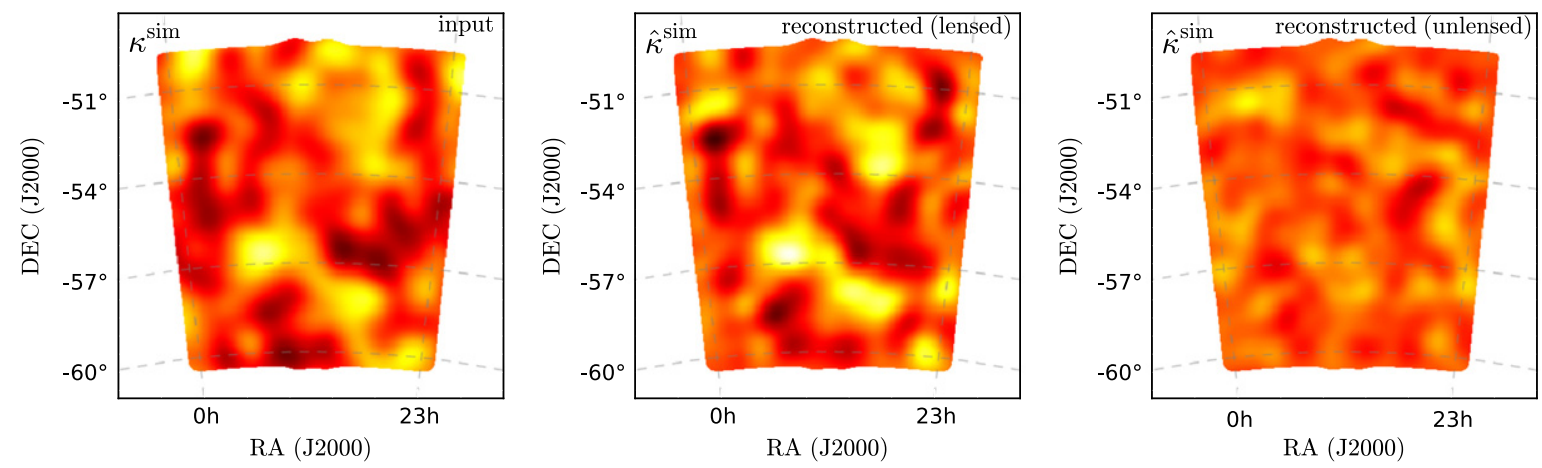

Figure 3. Example simulated $\kappa$-maps, plotted with the same color scale as Figure 2. Left: a simulated input $\kappa$-map. Middle: the reconstructed $\kappa$-map estimated from a noisy simulation that has been lensed using the potential shown in the left panel. Right: the reconstructed $\kappa$-map estimated from an unlensed simulation. Comparing the reconstructed lensed $\kappa$-map to the input map gives a visual sense of the fidelity of this reconstruction, and comparing to the unlensed $\kappa$-map gives a sense of the signal-to-noise in the MV $\kappa$-map.

significantly than the precision with which we measure the amplitude of the lensing spectrum $(14 \sigma$ versus $15 \% \sim 6.5 \sigma)$. This difference indicates that we measure the presence of lensing modes very significantly, however, the measurements are sample-variance dominated on large scales, limiting the precision of our constraint on the lensing power spectrum amplitude.

We plot the MV spectrum in comparison with other measurements in Figure 6. All spectra are visually consistent at the current sensitivity levels; we discuss these measurements in Section 8. We compare our MV spectrum amplitude directly to the most precise of these measurements (Planck) by recalculating the MV amplitude relative to the same fiducial spectrum used in Planck Collaboration XVII (2013). Planck measured an amplitude of $A_{\text {Planck }}=0.94 \pm 0.04$; we find an amplitude relative to the same cosmology of $A_{\mathrm{MV}}^{\text {Planck-Fiducial }}=0.90 \pm 0.16$, which is consistent.

\section{SYSTEMATIC ERROR CHECKS}

We perform a suite of tests for systematic errors in our data. For each test, we change one aspect of the analysis and recalculate the lensing spectrum $\left(C_{b}^{\phi \phi}\right.$ sys $)$ and amplitude
$\left(A_{\mathrm{MV}, \text { sys }}\right)$. We then calculate the difference between this spectrum (or amplitude) and the spectrum (or amplitude) from the baseline analysis (the baseline spectrum and amplitude are the MV results we report in Section 6). The differencespectrum $\Delta C_{b}^{\phi \phi}$ and difference-amplitude $\Delta A_{\mathrm{MV}}$ are the shifts induced in the spectrum and the amplitude, respectively, by the systematic under consideration; they can be expressed as

$$
\begin{gathered}
\Delta C_{b}^{\phi \phi}=C_{b, \mathrm{sys}}^{\phi \phi}-C_{b}^{\phi \phi} \\
\Delta A_{\mathrm{MV}}=A_{\mathrm{MV}, \mathrm{sys}}-A_{\mathrm{MV}} .
\end{gathered}
$$

The difference-spectra and difference-amplitudes are calculated for the data as well as for each simulation.

For each test, we use two metrics to determine if the data pass the test; these metrics are shown in Table 3. The first metric considers shifts in the lensing spectrum. We calculate the $\chi^{2}$ of the data difference-spectrum $\left(\Delta C_{b, \text { data }}^{\phi \phi}\right)$ using the variance of the simulation difference-spectra $\left(\sigma_{b, \text { sys }}\right)$ as the uncertainty. This can be expressed as

$$
\chi_{\text {sys }}^{2}=\sum_{b} \frac{\left(\Delta C_{b, d \text { data }}^{\text {d. }}\right)^{2}}{\sigma_{b, \text { sys }}^{2}} .
$$




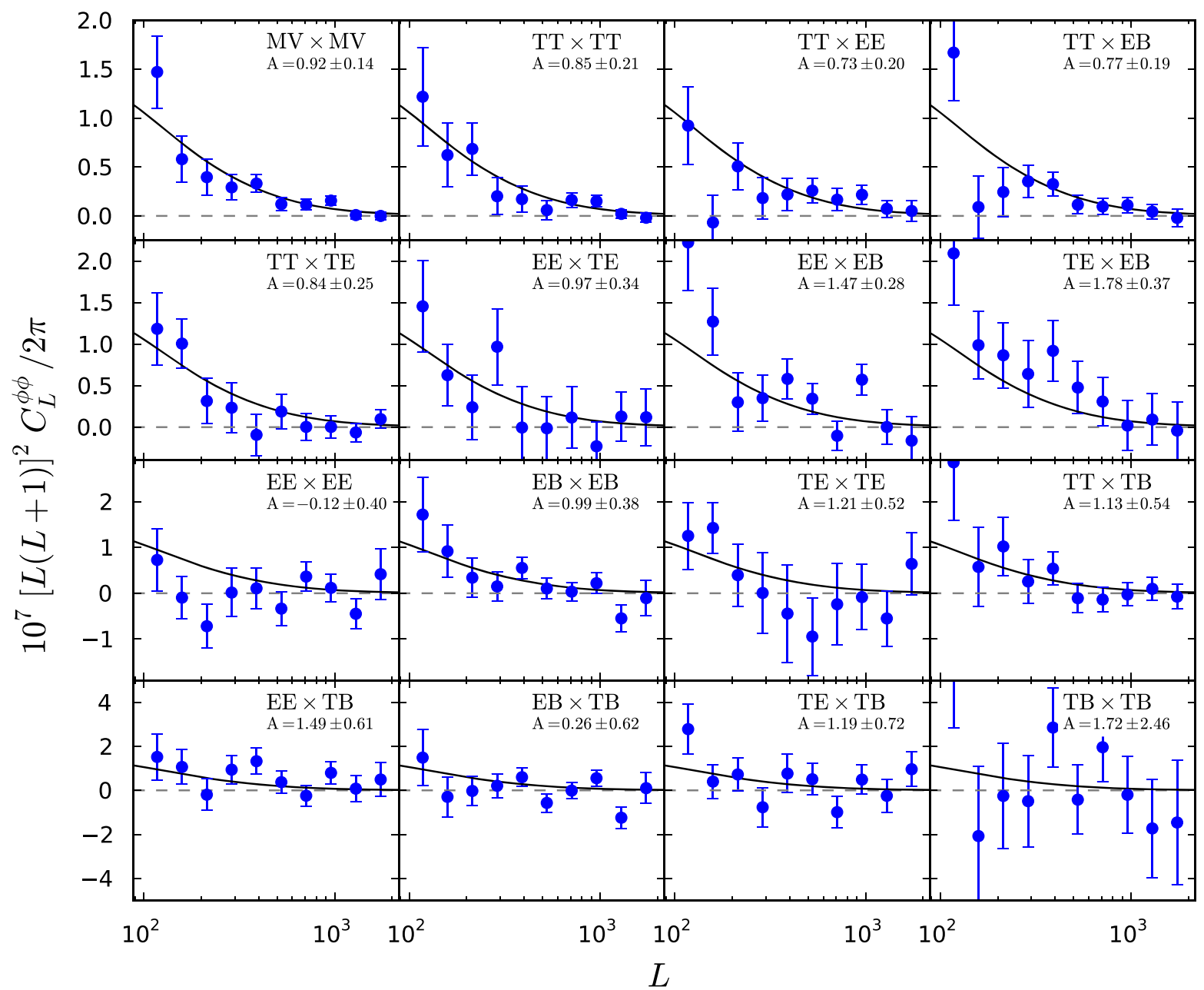

Figure 4. $\hat{C}_{L_{b}}^{\phi \phi}$ power spectra for all estimators we consider in this paper. "MV $\times \mathrm{MV}$ " is the spectrum from the minimum-variance estimator. The amplitudes of each spectrum relative to the fiducial PLANCK+Lens+WP+HIGHL model are calculated with Equation (27) and shown in each panel.

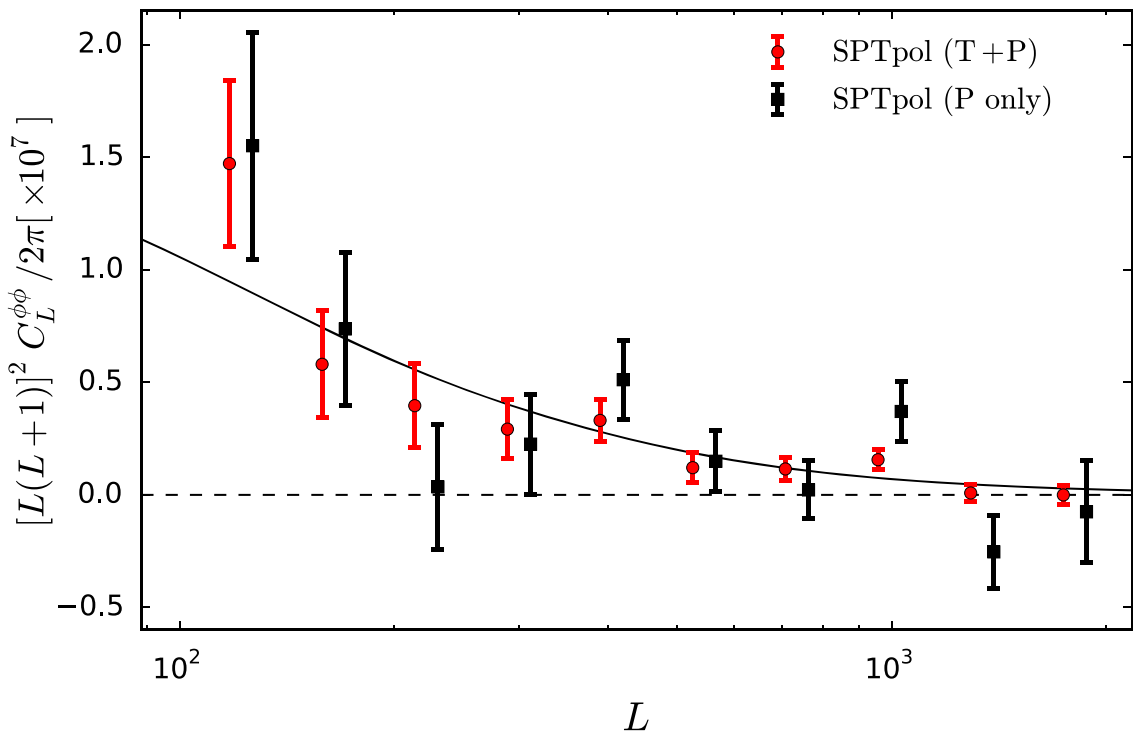

Figure 5. Lensing potential power spectrum bandpowers estimated from SPTpol. The MV and POL spectra are shown with red circles and black squares, respectively. The black solid line shows the PLANCK+LENS+WP+HIGHL best-fit $\Lambda$ CDM model. Note the POL points have been shifted by $1 / 4$ of a bin in $L$ for plotting purposes. 
Table 2

MV Lensing Bandpowers

\begin{tabular}{lccc}
\hline \hline$\left[L_{\min }\right.$ & $\left.L_{\max }\right]$ & $L_{b}$ & $10^{7}\left[L_{b}\left(L_{b}+1\right)\right]^{2} \hat{C}_{b}^{\phi \phi} / 2 \pi$ \\
\hline$[100$ & $133]$ & 117 & $1.47 \pm 0.37$ \\
{$[134$} & $181]$ & 158 & $0.58 \pm 0.24$ \\
{$[182$} & $244]$ & 213 & $0.40 \pm 0.19$ \\
{$[245$} & $330]$ & 288 & $0.29 \pm 0.13$ \\
{$[331$} & $446]$ & 389 & $0.331 \pm 0.092$ \\
{$[447$} & $602]$ & 525 & $0.121 \pm 0.067$ \\
{$[603$} & $813]$ & 708 & $0.115 \pm 0.053$ \\
{$[814$} & $1097]$ & 956 & $0.156 \pm 0.046$ \\
{$[1098$} & $1481]$ & 1290 & $0.008 \pm 0.038$ \\
{$[1482$} & $1998]$ & 1741 & $-0.000 \pm 0.041$ \\
\hline
\end{tabular}

Note. The bandpowers for the MV spectrum are presented here as defined in Equation (29) and shown in Figures 5 and 6. Bins are evenly spaced in $\log (L)$, and bandpowers are reported at the center of each bin

The probability-to-exceed (PTE) of this $\chi^{2}$ is calculated from a $\chi^{2}$ distribution with 10 degrees of freedom (corresponding to the 10 bins in our spectrum).

The second metric considers the change in the lensing amplitude. We calculate the lensing difference-amplitudes $\left(\Delta A_{\mathrm{MV}}\right)$ as defined in Equation (30) for the data and for each simulation. We then calculate the variance of the simulation difference-amplitudes $\left(\operatorname{var}\left(\Delta A_{\mathrm{MV} \text {,sim }}\right)\right)$; this variance estimates the expected magnitude of the change in the lensing amplitude. Specifically, we expect the magnitude of the lensing differenceamplitude in the data to be less than or equal to $\sqrt{\operatorname{var}\left(\Delta A_{\mathrm{MV} \text {,sim }}\right)}$ in $\sim 68 \%$ of similar measurements. Finally, we calculate the PTE of the data difference-amplitude directly from the simulations as the percentage of simulations that have a difference-amplitude with a larger magnitude than $\Delta A_{\mathrm{MV}}$ for the data. The data difference-amplitude, variance of simulated difference-amplitudes, and PTEs for each test are shown in Table 3.

The individual tests are described in detail below, and the results are reported in Table 3 and Figure 8.

1. $\ell_{\mathrm{xmin}}$ cut: As described in Section 2, we cut all modes with $\left|\ell_{x}\right|<450$ from the CMB maps. In this test, we adjust that cut from $\left|\ell_{x}\right|<450$ to 400 and 500 . When more $\ell$-space is removed by increasing the $\left|\ell_{x}\right|$ cut to 500 , the change in the lensing spectrum and amplitude are consistent with expectations from simulations. On the other hand, including the region between $400<\left|\ell_{x}\right|<450$ causes an unexpectedly large shift $(>2 \sigma)$ in the lensing amplitude, thus motivating the placement of this cut. Even in this case, however, the change in the amplitude is only $0.4 \sigma$ with respect to the statistical uncertainty on the lensing spectrum amplitude.

2. $\ell_{\max }$ cut: We adjust the maximum value of $\ell$ from $\mathrm{CMB}$ maps that is used in the estimator from $\ell<3000$ to 2500 and 3500. This corresponds to adjusting the upper bound of the integral in Equation (9). We find the data are consistent with the expectation from simulations in this test.

3. Apodized Mask: We apodize the sky and point-source mask with a cosine profile on the edges, and recalculate the amplitude and $C_{L}^{\phi \phi}$. The change in amplitude is consistent with the expectation from simulations.
4. C-inverse test: We recalculate the MV spectrum and amplitude using the diagonal approximation to the $C$ inverse filter (e.g., $\mathcal{F}_{\ell}^{X}$ from Equation (11)). The covariance of the simulations increases by a factor of four over the nominal covariance (due in part to the fact that the $C$-inverse filter effectively apodizes the map, while the diagonal approximation is just an inversevariance filter with no apodization), but the shift in the lensing amplitude is consistent with the shift of the simulations.

5. Curl test: We replace the gradient estimator with a curl estimator that is optimized for curl-like sources (Cooray et al. 2005). Specifically, we use the curl estimators specified in Table 3 of Namikawa et al. (2012). We estimate and remove the N0 contribution to the curl estimator as in Equation (22); thus the curl test provides a second check of the $\mathrm{N} 0$ estimation procedure. A non-zero curl signal would indicate contamination in the nominal gradient reconstruction from non-Gaussian secondary effects or foregrounds (Cooray et al. 2005). We calculate the curl signal, and find it to be consistent with the expectation from simulations in both the MV estimator $\left(\Delta A_{\mathrm{MV}}=0.082 \pm 0.092, C_{L}^{\phi \phi}\right.$ Spectrum PTE $\left.=0.69\right)$ and the POL estimator $\left(\Delta A_{\mathrm{POL}}=0.055 \pm 0.110, C_{L}^{\phi \phi}\right.$ Spectrum PTE $=0.52$ ).

6. Scan Direction: We perform a "jackknife" null test on the telescope scan direction. This test is sensitive to any systematic differences between left-going and right-going scans. We calculate null maps by subtracting all leftgoing scans from all right-going scans. The resulting maps should be free from signal but still contain any systematic difference between left-going and right-going maps. Because this is a null test, we calculate the pass-fail metrics for the jackknife spectrum and amplitude relative to zero (rather than relative to the MV spectrum and amplitude). Formally, this means replacing the baseline spectrum $C_{b}^{\phi \phi}$ and amplitude $A_{\mathrm{MV}}$ in Equation (30) with zeros, and using noise-only simulations. We find that the null spectrum and amplitude are consistent with noise.

Finally, we compare the spectra and amplitudes from each of the estimators with those from the MV spectrum. We find that the spectra are mostly consistent. The "TExEB" spectrum amplitude is high by $\sim 2.1 \sigma$ relative to the expectation from simulations, while the "EExEE" spectrum amplitude is low by $\sim 2.8 \sigma$. All other spectra are consistent to within $2 \sigma$.

\section{DISCUSSION}

This paper presents a measurement of the CMB lensing potential $\phi$ from $100 \mathrm{deg}^{2}$ of sky observed in temperature and polarization with SPTpol. Using a quadratic estimator analysis including polarization information, we have constructed a map of the lensing convergence field. Individual Fourier modes in this map are measured with signal-to-noise greater than one in the angular wavenumber or multipole range $100<L<250$. This represents the highest signal-to-noise map of the integrated lensing potential made from the CMB to date. The power spectrum of the lensing potential $C_{L}^{\phi \phi}$ was calculated from these maps. We have verified that this measurement is robust against systematics by performing a suite of systematics and null tests. 


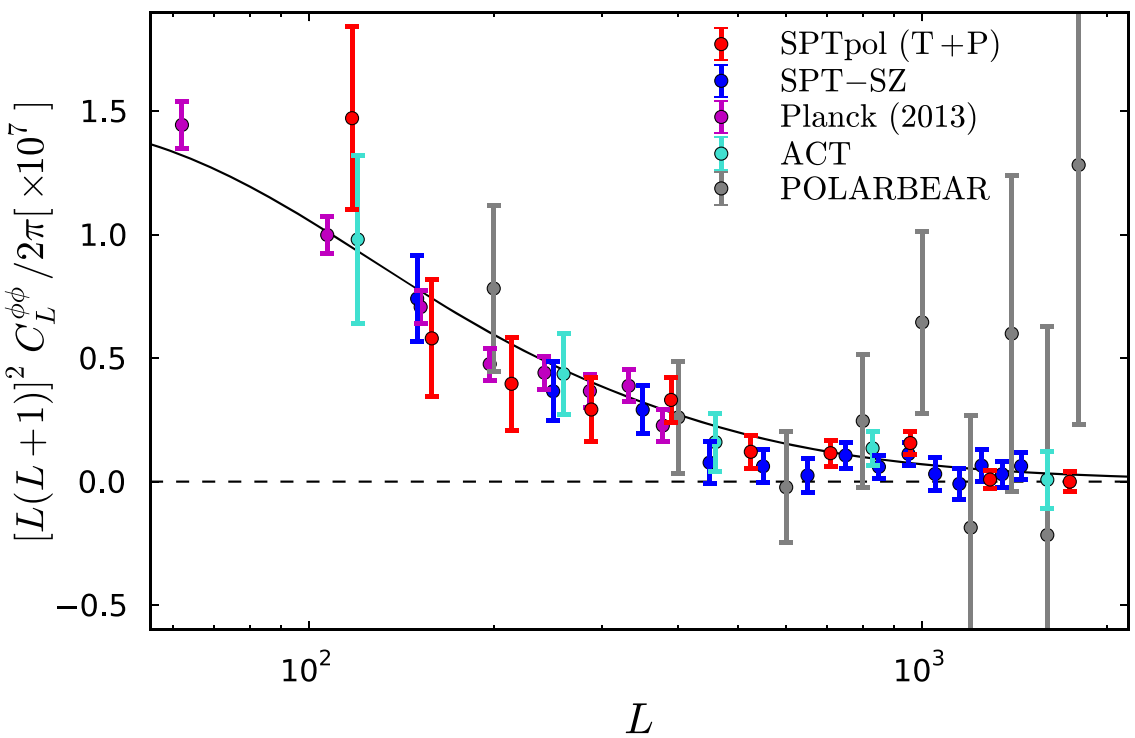

Figure 6. Lensing potential power spectrum bandpowers estimated from SPTpol, as well as those previously reported for temperature by SPT-SZ (van Engelen et al. 2012), ACT (Das et al. 2014), Planck (Planck Collaboration XVII 2013), and for polarization by POLARBEAR (POLARBEAR Collaboration 2014c). The black solid line shows the PLANCK+Lens+WP+HIGHL best-fit $\Lambda$ CDM model.

Table 3

MV Systematic Error and Null Tests

\begin{tabular}{l|rc|cc}
\hline \hline Test Name & \multicolumn{1}{|c|}{$\chi^{2}$} & $(\mathrm{PTE})$ & $\begin{array}{c}\Delta A_{\mathrm{MV}} \\
\pm \operatorname{var}\left(\Delta A_{\mathrm{MV}, \mathrm{sim}}\right)\end{array}$ & $(\mathrm{PTE})$ \\
\hline$L-R$ jackknife & 7.1 & $(0.72)$ & $0.0040 \pm 0.0052$ & $(0.45)$ \\
$l_{\mathrm{xmin}}=400$ & 17.5 & $(0.06)$ & $-0.063 \pm 0.030$ & $(0.025)$ \\
$l_{\mathrm{xmin}}=500$ & 10.7 & $(0.38)$ & $0.053 \pm 0.033$ & $(0.10)$ \\
$l_{\max }=2500$ & 13.6 & $(0.19)$ & $-0.122 \pm 0.107$ & $(0.26)$ \\
$l_{\max }=3500$ & 9.2 & $(0.51)$ & $0.007 \pm 0.067$ & $(0.91)$ \\
Apodized Mask & 13.6 & $(0.19)$ & $-0.043 \pm 0.034$ & $(0.22)$ \\
$C$-inverse & 9.6 & $(0.47)$ & $0.146 \pm 0.534$ & $(0.78)$ \\
Curl & 7.4 & $(0.69)$ & $0.082 \pm 0.092$ & $(0.39)$ \\
\hline
\end{tabular}

Note. Results of systematics tests. For each test, the $\chi^{2}$ and PTE of the $C_{L}^{\phi \phi}$ spectrum are shown in the second column. The change in amplitude and associated PTE are shown in the third column. See Equation (30) for more detail. The $l_{\mathrm{xmin}}=400$ test fails, which is why we place the cut higher, at $l_{\mathrm{xmin}}=450$ in the analysis.

We compare this measurement to a fiducial spectrum taken from the PLANCK+Lens+WP+HIGHL best-fit $\Lambda$ CDM model and find a relative amplitude of $A_{\mathrm{MV}}=0.92 \pm 0.14$ (Stat.) \pm 0.08 (Sys.). This corresponds to a $18 \%$ measurement of the amplitude. This measurement rejects the no-lensing hypothesis at $14 \sigma$. If instead only polarized estimators are used, we find $A_{\mathrm{POL}}=0.92 \pm 0.24$ (Stat.) \pm 0.11 (Sys.). This is a $29 \%$ measurement of the amplitude. This measurement rejects the no-lensing hypothesis using polarization information only at 5.9 $\sigma$. The MV and POL amplitudes are consistent at $0.47 \sigma$ and $0.32 \sigma$, respectively, with the best-fit $\Lambda$ CDM cosmology of the Planck+Lens+WP+highL dataset. The PTE of the $\chi^{2}$ relative to this theory spectrum for the MV and POL spectra are 0.49 and 0.10 , respectively. The PTEs for all individual estimators are nominal, with a max/min values of 0.96 and 0.16 , respectively. Of note, the bandpowers are consistent with the $\Lambda \mathrm{CDM}$ theory prediction even at high $L$, where nonlinear structure growth or contamination from foregrounds are more relevant.

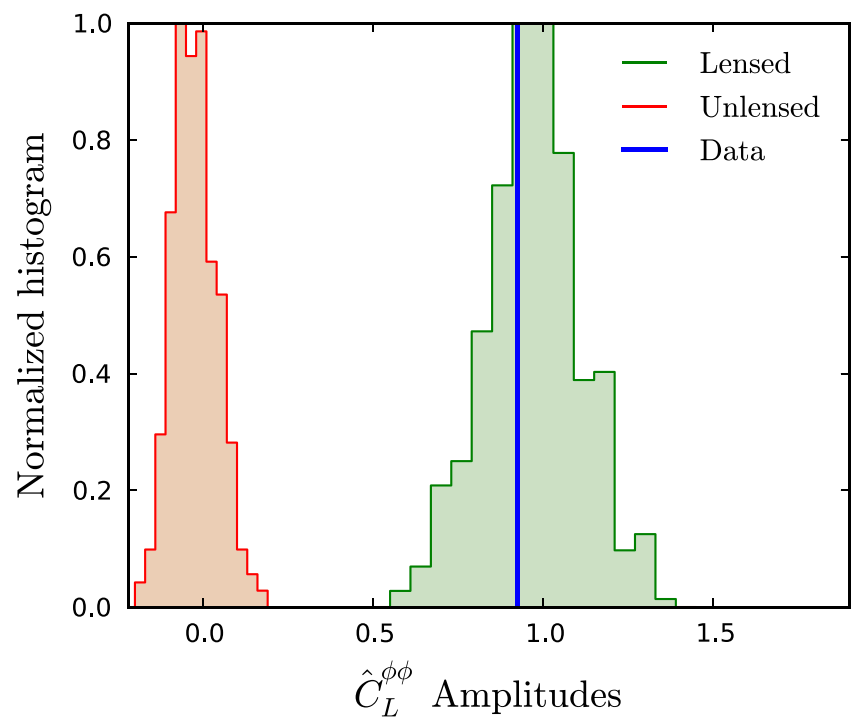

Figure 7. Distribution of reconstructed MV lensing amplitudes from simulations are shown here for lensed (green) and unlensed (red) simulations. The amplitude of the MV estimate for the data is shown as a blue line. The statistical uncertainty of the MV lensing construction is given by the standard deviation of the lensed simulations $\left(\Delta A_{\mathrm{MV}}=0.14\right)$. The significance with which we rule out the no-lensing hypothesis is calculated from the standard deviation of the unlensed simulations (0.065).

We also compare the amplitude of our measured lensing spectrum relative to other cosmologies. Within the context of a given cosmological model, the true amplitude of the lensing spectrum is by definition unity; any statistically significant deviation of the measured value of $A_{\text {lens }}$ from unity indicates tension with the assumed cosmological model. Replacing our fiducial lensing spectrum with the lensing spectrum derived from the $\Lambda$ CDM model that best fits the WMAP9+SPT-SZ dataset (a combination of WMAP9 data Hinshaw et al. 2013 and temperature power spectrum from Story et al. 2013), the best-fit amplitude is $A_{\mathrm{MV}}^{\mathrm{WMAP} 9+\mathrm{SPT}}=1.05$. This represents a shift of $\sim 13 \%$, or $\sim 1 \sigma$ (statistical-only) from the best-fit value assuming our fiducial cosmology. The current SPTpol 

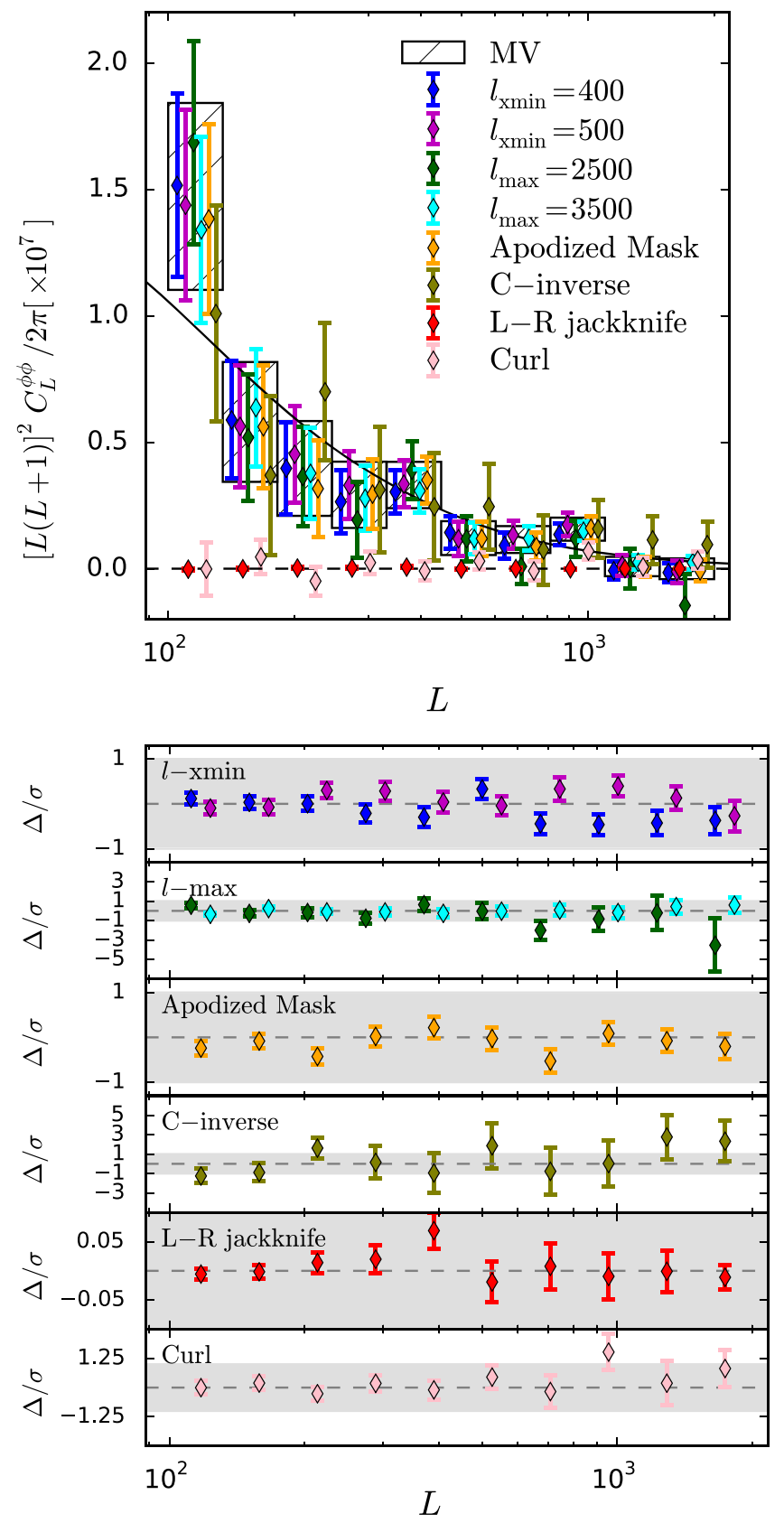

Figure 8. Power spectrum consistency tests. The upper panel shows the spectrum of each consistency test. The lower panels show the same set of consistency tests, plotted as the difference between each consistency test relative to the fiducial spectrum, divided by the $1 \sigma$ statistical error bar of the MV spectrum. Note that the statistical uncertainty on these difference-spectra will be a function of the analysis and not necessarily the same as for the baseline MV spectrum. The gray band in each of the lower panels shows the $1 \sigma$ statistical uncertainty region. The error bars shown in the lower panels are calculated from the variance in simulations of each consistency test.

measurement is consistent with the predictions of both cosmologies and cannot distinguish between them.

In comparison with previous quadratic estimator measurements from SPT and ACT, which used roughly six times the sky area $\left(\sim 600 \mathrm{deg}^{2}\right)$, we have measured the lensing potential with similar precision ${ }^{43}: 18 \%$ in this measurement, as compared

\footnotetext{
$\overline{43}$ The fractional precision is reported relative to the mean of each measurement.
}

to $19 \%$ from van Engelen et al. (2012) and 22\% from Das et al. (2014). The precision of our measurement, however, is limited by sample-variance of the lenses themselves; as a result, our measurement rules out the no-lensing hypothesis much more significantly, at $14 \sigma$ in our MV measurement. For comparison, no lensing was ruled out at $6.3 \sigma$ in van Engelen et al. (2012) (although the sample variance was scaled as a function of $A_{L}$ in that calculation) and at $>4.6 \sigma$ in Das et al. (2014) (where sample variance was included). The temperature measurement from Planck is derived from over $70 \%$ of the sky and thus has much lower sample variance. At $25 \sigma$ it is highly significant, although no modes are measured with a signal-to-noise ratio greater than one (Planck Collaboration XVII 2013). Our measurement is consistent with that from Planck; we calculate the MV amplitude relative to the same fiducial cosmology used in Planck Collaboration XVII (2013), and find an amplitude of $A_{\mathrm{MV}}^{\text {Planck-Fiducial }}=0.90 \pm 0.16$, which is consistent with the measurement from Planck of $A_{\text {Planck }}=0.94 \pm 0.04$. Finally, our polarization-only measurement improves on the sensitivity achieved by POLARBEAR: the SPTpol POL spectrum rules out no-lensing at $5.9 \sigma$, as compared to $4.2 \sigma$ from (POLARBEAR Collaboration 2014c).

Because of the high signal-to-noise of the SPTpol measurement presented here and the fact that the BICEP2 and KECK Array experiments have observed the same patch of sky, this SPTpol mass map will be powerful for de-lensing primordial BB power spectrum measurements. Additionally, these deep sptpol mass maps will enable significant cross-correlation measurements with other tracers of large-scale structure.

Polarization measurements will continue to improve rapidly. SPTpol will soon start its fourth year of observing a larger $500 \mathrm{deg}^{2}$ patch of sky, ACTpol and POLARBEAR observations are continuing, and Planck is expected to release a similar polarized analysis in the near future. With these and the next generation of $\mathrm{CMB}$ polarization experiments being planned (e.g., SPT3G (Benson et al. 2014), AdvancedACTpol (Calabrese et al. 2014), Simons Array (Arnold et al. 2014)), CMB lensing will become an exceptionally powerful probe of structure evolution in the universe.

The South Pole Telescope program is supported by the National Science Foundation through grant PLR-1248097. Partial support is also provided by the NSF Physics Frontier Center grant PHY-0114422 to the Kavli Institute of Cosmological Physics at the University of Chicago, the Kavli Foundation, and the Gordon and Betty Moore Foundation through Grant GBMF\#947 to the University of Chicago. The McGill authors acknowledge funding from the Natural Sciences and Engineering Research Council of Canada, Canadian Institute for Advanced Research, and Canada Research Chairs program. The CU Boulder group acknowledges support from NSF AST-0956135. J. W. H. is supported by the National Science Foundation under Award No. AST-1402161. B. B. is supported by the Fermi Research Alliance, LLC under Contract No. De-AC02-07CH11359 with the U.S. Department of Energy. TdH is supported by a Miller Research Fellowship. This work is also supported by the U.S. Department of Energy. Work at Argonne National Lab is supported by UChicago Argonne, LLC, Operator of Argonne National Laboratory (Argonne). Argonne, a U.S. Department of Energy Office of Science Laboratory, is operated under Contract No. DE-AC0206CH11357. We also acknowledge support from the Argonne Center for Nanoscale Materials. This research used resources of 
the Calcul Quebec computing consortium, part of the Compute Canada network, and of the National Energy Research Scientific Computing Center, a DOE Office of Science User Facility supported by the Office of Science of the U.S. Department of Energy under Contract No. DE-AC02-05CH11231. The data analysis pipeline uses the scientific Python stack (Jones et al. 2001; Hunter 2007; van der Walt et al. 2011) and the HDF5 file format (The HDF Group 1997).

\section{REFERENCES}

Arnold, K., Stebor, N., Ade, P. A. R., et al. 2014, Proc. SPIE, 9153, 1 Austermann, J. E., Aird, K. A., Beall, J. A., et al. 2012, Proc. SPIE, 8452, 1 Benson, B. A., Ade, P. A. R., Ahmed, Z., et al. 2014, Proc. SPIE, 9153, 1 BICEP2 Collaboration 2014, PhRvL, 112, 241101

BICEP2/Keck and Planck Collaborations, Ade, P. A. R., et al. 2015, arXiv: 1502.00612

Blanchard, A., \& Schneider, J. 1987, A\&A, 184, 1

Calabrese, E., Cooray, A., Martinelli, M., et al. 2009, PhRvD, 80, 103516

Calabrese, E., Hložek, R., Battaglia, N., et al. 2014, JCAP, 8, 10

Carlstrom, J. E., Ade, P. A. R., Aird, K. A., et al. 2011, PASP, 123, 568

Cole, S., \& Efstathiou, G. 1989, MNRAS, 239, 195

Cooray, A., Kamionkowski, M., \& Caldwell, R. R. 2005, PhRvD, 71, 123527

Crites, A. T., Henning, J. W., Ade, P. A. R., et al. 2014, arXiv:1411.1042

Das, S., Louis, T., Nolta, M. R., et al. 2014, JCAP, 4, 14

Górski, K. M., Hivon, E., Banday, A. J., et al. 2005, ApJ, 622, 759

Hanson, D., et al. 2013, PhRvL, 111, 141301

Hinshaw, G., Larson, D., Komatsu, E., et al. 2013, ApJS, 208, 19

Hirata, C. M., Ho, S., Padmanabhan, N., Seljak, U., \& Bahcall, N. A. 2008, PhRvD, 78, 043520

Hu, W. 2001, ApJL, 557, L79

Hu, W., \& Okamoto, T. 2002, ApJ, 574, 566
Hu, W., \& White, M. 1997, NewA, 2, 323

Hunter, J. D. 2007, CSE, 9, 90

Jones, E., Oliphant, T., Peterson, P., et al. 2001, SciPy: Open source scientific tools for Python

Keisler, R., Hoover, S., Harrington, N., et al. 2015, arXiv:1503.02315

Kesden, M., Cooray, A., \& Kamionkowski, M. 2003, PhRvD, 67, 123507

Lesgourgues, J., \& Pastor, S. 2006, PhR, 429, 307

Lewis, A. 2005, PhRvD, 71, 083008

Lewis, A., \& Bridle, S. 2002, PhRvD, 66, 103511

Lewis, A., \& Challinor, A. 2006, PhR, 429, 1

Lewis, A., Challinor, A., \& Hanson, D. 2011, JCAP, 3, 18

Mocanu, L. M., Crawford, T. M., Vieira, J. D., et al. 2013, ApJ, 779, 61

Namikawa, T., Hanson, D., \& Takahashi, R. 2013, MNRAS, 431, 609

Namikawa, T., Yamauchi, D., \& Taruya, A. 2012, JCAP, 1, 7

Osborne, S. J., Hanson, D., \& Doré, O. 2014, JCAP, 3, 24

Planck Collaboration XVI 2014, A\&A, 571, A16

Planck Collaboration XVII 2013, A\&A, 517, A17

POLARBEAR Collaboration 2014a, ApJ, 794, 171

POLARBEAR Collaboration 2014b, PhRvL, 112, 131302

POLARBEAR Collaboration 2014c, PhRvL, 113, 021301

Seljak, U. 1996, ApJ, 463, 1

Seljak, U., \& Zaldarriaga, M. 1999, PhRvL, 82, 2636

Shaw, L. D., Nagai, D., Bhattacharya, S., \& Lau, E. T. 2010, ApJ, 725,1452

Smith, K. M., Zahn, O., \& Doré, O. 2007, PhRvD, 76, 043510

Story, K. T., Reichardt, C. L., Hou, Z., et al. 2013, ApJ, 779, 86

The HDF Group 1997, Hierarchical Data Format, version 5, http://www. hdfgroup.org/HDF5/

van der Walt, S., Colbert, S., \& Varoquaux, G. 2011, CSE, 13, 22

van Engelen, A., Bhattacharya, S., Sehgal, N., et al. 2014a, ApJ, 786, 13

van Engelen, A., Bhattacharya, S., Sehgal, N., et al. 2012, ApJ, 756, 142

van Engelen, A., Sherwin, B. D., Sehgal, N., et al. 2014b, arXiv:1412.0626

Vanderlinde, K., Crawford, T. M., de Haan, T., et al. 2010, ApJ, 722, 1180

Zaldarriaga, M., \& Seljak, U. 1998, PhRvD, 58, 23003 